\title{
Computation and analysis of bound vibrational spectra of the Neon tetramer using row orthonormal hyperspherical coordinates.
}

\author{
Bruno Lepetit* \\ Laboratoire Collisions, Agrégats, Réactivité (UMR 5589, \\ CNRS-Université Paul Sabatier Toulouse 3) IRSAMC, 31062 Toulouse Cedex 9, France
}

(Dated: August 18, 2020)

\begin{abstract}
The paper presents the first implementation of the row-orthonormal hyperspherical coordinate formalism for the computation of the vibrational spectrum of a tetratomic system. The wavefunction of $\mathrm{Ne}_{4}$ is expanded on a large basis set of hyperspherical harmonics generated numerically. This method not only provides spectra with reasonable accuracy, but also gives physical insight into the vibrational dynamics of the system. The characteristics of the spectra are related to the symmetry and localization of the wavefunction in configuration space.
\end{abstract}

PACS numbers:

Keywords:

*Electronic address: bruno.lepetit@irsamc.ups-tlse.fr 


\section{INTRODUCTION}

The hyperspherical coordinate method has been used extensively over the past decades to study few-body problems. It relies on the parametrization of a N-body system with a hyperradius $\rho$ which gives to the global size of the system and a set of 3N-4 angles which describe the shape of the system. Since the method has the capability to describe the fragmentation channels of the system, it has been used extensively for collisional problems which involve continuum states, like reactive scattering (for example, ref. 1, 2), ultracold physics [3], high energy nuclear collisions [4], as well as for bound state computations, for instance in atomic clusters [5] or nuclei [4]. It is surprising however that an overwhelming majority of these works concerns 3-body problems. This is an indication that it remains even today technically challenging to deal with 4 bodies without drastic approximations. Yet, on rare occasions like the study of 4-body recombinations, the use of such methods was an essential ingredient to achieve significant progress in our understanding of ultra-cold fermionic [6] and bosonic [3, 7] physics.

In the field of rare gas clusters $\operatorname{Rg}_{N}$ also, almost all studies of (ro-)vibrational spectra concern trimers (for a review, ref. 5). As an exception, a pioneering study [8, 9] of the vibrational spectra for $\mathrm{Rg}=\mathrm{He}, \mathrm{Ne}$, Ar was performed using hyperspherical coordinates for $\mathrm{N}$ up to $\mathrm{N}=6$, but within the frame of the drastic adiabatic approximation which restricts the validity of the results at most to a very limited number of low lying states. However, this study provided useful qualitative insight in the cluster dynamics. In fact, the only two accurate computations of (ro-)vibrational spectra for rare gas clusters beyond trimers which we are aware of were obtained using Jacobi coordinates and potential-optimized discrete variable representation [10] functions. The first one is the vibrational spectrum computation of $\mathrm{Ar}_{4}$ described in ref. 11, the second one the full rovibrational spectrum computation of $\mathrm{Ne}_{4}$ described in ref. 12. The recent availability of these latter $\mathrm{Ne}_{4}$ spectra provides a good opportunity to test for the first time the capability of the hyperspherical coordinate method to obtain the vibrational spectrum of a tetratomic system beyond the drastic adiabatic approximation.

The hyperspherical harmonics [13] are eigenfunctions of the fixed-hyperradius kinetic energy operator and form an orthogonal basis in which the complicated angular part of the kinetic energy operator has a very simple diagonal matrix representation. They can be used 
in 2 ways in the expansion of the wavefunction of the system [14]. One way popular in nuclear sciences [4] is to combine them with hyperradial functions in product functions on which the total wavefunction of the system is expanded directly. Another way popular in chemical physics is to use them to expand the eigenfunctions of the fixed $\rho$-Hamiltonian which are called channel functions [6] or surface functions or, following ref. 15, local-hypersphericalsurface-functions (LHSF). These LHSF form a compact basis set on which to expand the total wavefunction of the system. This set of surface functions provides a hyperspherical adiabatic representation of the system containing useful physical information on its dynamical properties. The hyperspherical adiabatic representation becomes a hyperspherical adiabatic approximation $[8,9]$ when a single surface function is kept in the expansion of the full wavefunction. In the present paper, we will show how analysis of the surface functions and of their $\rho$-dependent energies provides insight into the vibrational dynamics of the $\mathrm{Ne}_{4}$ cluster. In particular, we will identify the pyramid, rhombus and trigonal geometries which play an important role. For completeness, we note that correlated Gaussians have been used as a successful alternative to hyperspherical harmonics in the expansion of LHSF [6, 14].

There are 2 types of hyperspherical coordinates and harmonics. The most standard one is the Delves type $[6,16,17]$ obtained from an extension of the vector spherical representation to a set of several Jacobi vectors associated to a given clustering scheme of the particles. The corresponding hyperspherical harmonics are known analytically [6, 13]. They may not be always the optimum choice of coordinates in practical applications. Their angles are defined with respect to a space fixed frame and do not take advantage of the isotropy of space, as Euler angles would. Furthermore, an identical particle symmetrization operation is difficult to implement in this coordinate system because it potentially changes all angular coordinates [18]. Consequently, giving up implementing permutations on the wavefunction may be preferred in some applications even if the price to pay is larger computations [19]. In order to overcome these difficulties, symmetrized coordinates, which we call, following ref. 20, row-orthonormal-hyperspherical coordinates (ROHC), were developped in ref. 20, 21 following the pioneering work of ref. 22, 23. In this coordinate system, the 9 dimensional parameter space can be considered as the superposition of 3 subspaces. One is generated by the 3 Euler angles, which define the orientation in space of a body frame tied to the principal axes of inertia of the molecular system. The second subspace is the 3-dimensional kinematic invariant subspace [24] $\mathcal{S}^{\mathcal{K} \mathcal{I}}$. It contains the hyperradius $\rho$ and 2 angles which provide a 
parametrization of the 3 moments of inertia of the system. Finally, the kinematic rotation subspace $\mathcal{S}^{\mathcal{K R}}$ is defined by 3 angles which parametrize the shape of the system. This coordinate system has distinctive advantages. The Euler angles allow to take full advantage of the isotropy of space and when the total angular momentum of the system is 0 , the wavefunction becomes independent of them. The effects on coordinates of identical particle permutations is confined to $\mathcal{S}^{\mathcal{K} R}$, which makes their implementation in the wavefunction easier. On the other hand, the hyperspherical harmonics are more difficult to express in ROHC than in Delves' coordinates. In early works, only a limited number of harmonics could be obtained analytically [22-26]. A general recursion relation was implemented in a mathematical formal calculation program to enlarge the set of harmonics known analytically [15], at the price of practical difficulties. For instance, the recursion provides over-complete sets of linearly-dependent harmonics which have to be culled to reduce them to linearlyindependent sets using a formal but computationally intensive algorithm. An alternative generation approach entirely numerical was therefore proposed in [27], it relies on a selection of simple trigonometric basis functions in which to expand the hyperspherical harmonics according to their symmetry properties. However, the resulting harmonics have never been used in physical applications.

Therefore, the present paper represents to our knowledge the first successful use of 4-body ROHC harmonics to a molecular problem. It presents the results of the computation of the vibrational spectrum of the Neon tetramer using these symmetrized hyperspherical coordinates and harmonics. Section II presents the formalism, first the coordinates (subsection II A), then the harmonics used to expand the LHSF (subsection II B) and the full wavefunction expansion on the LHSF (subsection IIC). Subsection IID provides technical details on our implementation of the $\mathrm{Ne}_{4}$ problem. In section III, we first present the obtained vibrational spectra and we discuss their accuracy by comparison with the reference data of ref. [12]. Then, we describe the physical insight provided by the hyperspherical method : we present the LSHF energy curves as a function of $\rho$ (subsection III B), the LHSF themselves (subsection III C) and the bound states (subsection III D). 


\section{METHOD}

\section{A. Coordinates and kinetic energy}

The ROHC formalism used in the present paper have been described previously $[15,20,27]$. We summarize here important information and refer the reader to these papers for further details. Let $\boldsymbol{\rho}^{s f}$ be the Jacobi matrix, whose columns are the three cartesian coordinates in an arbitrary space-fixed frame of the three mass-scaled Jacobi vectors $\left(\mathbf{r}^{(1)}, \mathbf{r}^{(2)}, \mathbf{r}^{(3)}\right)$ associated to a given clustering scheme of the 4 particles which we choose as follows : attributing arbitrarily numbers 1-4 to the 4 identical neon atoms, $\mathbf{r}^{(1)}$ connects atoms 1 and 2, $\mathbf{r}^{(2)}$ atom 3 to the center of mass of atoms 1 and 2, $\mathbf{r}^{(3)}$ atom 4 to the center of mass of the 3 others. The ROHC $\chi, \rho, \mathbf{a}, \boldsymbol{\delta}$ associated to this clustering scheme are defined implicitely by :

$$
\boldsymbol{\rho}^{s f}=(-1)^{\chi} \tilde{\mathbf{R}}(\mathbf{a}) \rho \mathbf{N}(\theta, \phi) \tilde{\mathbf{R}}(\boldsymbol{\delta})
$$

$\chi$ is the chirality variable, a refers collectively to the three Euler angles $(a, b, c)$ of the principal axes of inertia frame and $\rho$ is the hyperradius which, together with the angles $\theta, \phi$, parametrizes the three principal moments of inertia of the system and defines the kinematic invariant space $\mathcal{S}^{\mathcal{K I}}$. Three additional angles $\left(\delta_{1}, \delta_{2}, \delta_{3}\right)$ collectively denoted by $\boldsymbol{\delta}$ parametrize the kinematic rotation space $\mathcal{S}^{\mathcal{K} \mathcal{R}}$. The matrix $\tilde{\mathbf{R}}(\mathbf{a})$ is the transpose of the rotation matrix defined by [28] :

$$
\mathbf{R}(\mathbf{a})=\mathbf{M}_{\mathbf{1}}(c) \mathbf{M}_{\mathbf{2}}(b) \mathbf{M}_{\mathbf{1}}(a)
$$

where :

$$
\mathbf{M}_{1}(\omega)=\left(\begin{array}{ccc}
\cos \omega & \sin \omega & 0 \\
-\sin \omega & \cos \omega & 0 \\
0 & 0 & 1
\end{array}\right)
$$

and :

$$
\mathbf{M}_{\mathbf{2}}(\omega)=\left(\begin{array}{ccc}
\cos \omega & 0 & -\sin \omega \\
0 & 1 & 0 \\
\sin \omega & 0 & \cos \omega
\end{array}\right)
$$


These definitions are also valid for $\tilde{\mathbf{R}}\left(\delta_{1}, \delta_{2}, \delta_{3}\right)$. The matrix $\mathbf{N}(\theta, \phi)$ is a diagonal matrix, with diagonal elements :

$$
N_{11}(\theta, \phi)=\sin \theta \cos \phi, N_{22}(\theta, \phi)=\sin \theta \sin \phi, N_{33}(\theta, \phi)=\cos \theta
$$

Equation 1 is called the row-orthonormal form of the Jacobi matrix $\boldsymbol{\rho}^{s f}$ because matrix $\tilde{\mathbf{R}}(\boldsymbol{\delta})$ is row-orthonormal. In the present 4-particle case, the matrix $\tilde{\mathbf{R}}(\boldsymbol{\delta})$ is square and thus also column-orthonormal.

It was previously shown [20] that a one to one correspondence can be achieved between physical configurations and coordinate space by restricting the hyperspherical angles to :

$$
\begin{gathered}
0 \leq a, c<2 \pi, 0 \leq b \leq \pi \\
0 \leq \delta_{1}, \delta_{3}<\pi, 0 \leq \delta_{2} \leq \pi \\
0 \leq \phi \leq \pi / 4, \quad 0 \leq \theta \leq \theta_{M}=\arcsin \left[1 /\left(1+\cos ^{2} \phi\right)^{1 / 2}\right] \leq \arcsin (2 / 3)^{1 / 2} \simeq 54.7^{\circ}
\end{gathered}
$$

and $\chi=0$ or 1 .

In terms of these ROHC, the kinetic energy operator associated to the motion of the 4 nuclei is given by [27] :

$$
\hat{T}=-\frac{\hbar^{2}}{2 \mu} \frac{1}{\rho^{8}} \frac{\partial}{\partial \rho} \rho^{8} \frac{\partial}{\partial \rho}+\frac{\hat{\Lambda}^{2}(\boldsymbol{a}, \theta, \phi, \boldsymbol{\delta})}{2 \mu \rho^{2}}
$$

where $\mu=m / 4^{\frac{1}{3}}$ is the 4 -body reduced mass ( $m$ : Ne mass) of the system and $\hat{\Lambda}$ the grandcanonical angular momentum operator. Its complicated expression in terms of the eight internal hyperangles is given in ref. 27 , eq. 17, 19-25. It involves the nuclear motion angular momentum operator $\hat{J}$ as well as $\hat{L}$, a $\boldsymbol{\delta}$-dependent internal angular momentum operating in $\mathcal{S}^{\mathcal{K} R}$.

\section{B. Hyperspherical harmonics}

Since the 6 operators $\hat{\Lambda}^{2}, \hat{J}^{2}, \hat{J}_{z}$ (the component of $\hat{J}$ on the space-fixed $z$ axis), $\hat{L}^{2}, \hat{L}_{3}$ (the 3rd body-fixed type component of $\hat{L}$ ) and $\hat{I}$ (inversion of the Jacobi vectors $\mathbf{r}^{(1)}, \mathbf{r}^{(2)}, \mathbf{r}^{(3)}$ to their opposites, which, according to eq. 1, means that the chirality coordinate changes according to : $\chi \leftrightarrow \chi \pm 1$, while the other 9 coordinates are unchanged) commute with each 
other, we can find functions $F_{M_{J} M_{L}}^{n J} \underset{d}{L}$, the hyperspherical harmonics, which are simultaneous eigenfunctions of those 6 operators :

$$
\begin{aligned}
& \hat{\Lambda}^{2} F_{M_{J} M_{L} d}^{n J} \underset{d}{L}(\chi, \boldsymbol{a}, \theta, \phi, \boldsymbol{\delta})=n(n+7) \hbar^{2} F_{M_{J} M_{L}}^{J_{d}^{L}} \underset{D}{D}(\chi, \boldsymbol{a}, \theta, \phi, \boldsymbol{\delta}) \\
& \hat{J}^{2} F_{M_{J} M_{L}}^{{ }^{L}} \underset{d}{D}(\chi, \boldsymbol{a}, \theta, \phi, \boldsymbol{\delta})=J(J+1) \hbar^{2} F_{M_{J} M_{L} d}^{n{ }_{d}^{L}}(\chi, \boldsymbol{a}, \theta, \phi, \boldsymbol{\delta}) \\
& \hat{J}_{z} F_{M_{J} M_{L_{\lambda}} d \underset{d}{L}}^{D}(\chi, \boldsymbol{a}, \theta, \phi, \boldsymbol{\delta})=M_{J} \hbar F_{M_{J} M_{L_{\lambda}} d}^{\stackrel{D}{L}}(\chi, \boldsymbol{a}, \theta, \phi, \boldsymbol{\delta}) \\
& \hat{L}^{2} F_{M_{J} M_{L_{\lambda}}{ }^{J}}^{L}(\chi, \boldsymbol{a}, \theta, \phi, \boldsymbol{\delta})=L(L+1) \hbar^{2} F_{M_{J} M_{L} d}^{{ }_{L}^{L}} \underset{d}{D}(\chi, \boldsymbol{a}, \theta, \phi, \boldsymbol{\delta}) \\
& \hat{L}_{3} F^{n J}{ }_{M_{J} M_{L} d}^{L} \underset{d}{D}(\chi, \boldsymbol{a}, \theta, \phi, \boldsymbol{\delta})=M_{L} \hbar F_{M_{J} M_{L} d}^{L} \underset{d}{D}(\chi, \boldsymbol{a}, \theta, \phi, \boldsymbol{\delta})
\end{aligned}
$$

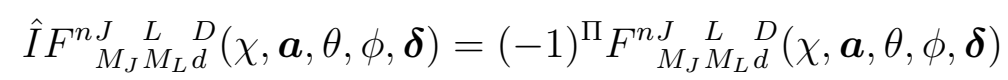

The quantum numbers $n, J, M_{J}, L, M_{L}$, $\Pi$ appearing in these expressions are all integers, satisfying the constraints :

$$
\begin{gathered}
n \geq 0 \quad 0 \leq J, L \leq n \\
-J \leq M_{J} \leq J \quad-L \leq M_{L} \leq L \\
\Pi=0 \text { or } 1
\end{gathered}
$$

The inversion quantum number $\Pi$ is implicitely contained in the principal quantum number $n$ through the relation : $(-1)^{\Pi}=(-1)^{n}$. The sub- and super-scripts $d$ and $D$ indicate that the $F$ functions can be degenerate, i.e. for a given set of quantum numbers $n, J, M_{J}, L$, $M_{L}$, there are $D$ linearly independent $F$ functions. The subscript $d$ indicates which of those degenerate functions is being considered.

These functions can be expanded on symmetrized Wigner functions defined by :

$$
\begin{aligned}
& \mathcal{D}_{M_{J} \Omega_{J}}^{J, \eta}(\boldsymbol{a})=\frac{1}{\sqrt{2\left(1+\delta_{\Omega_{J} 0}\right)}}\left(D_{M_{J} \Omega_{J}}^{J}(\boldsymbol{a})+\eta D_{M_{J}-\Omega_{J}}^{J}(\boldsymbol{a})\right) \\
& \mathcal{D}_{\Omega_{L} M_{L}}^{L, \eta}(\boldsymbol{\delta})=\frac{1}{\sqrt{2\left(1+\delta_{\Omega_{L} 0}\right)}}\left(D_{\Omega_{L} M_{L}}^{L}(\boldsymbol{\delta})+\eta D_{-\Omega_{L} M_{L}}^{L}(\boldsymbol{\delta})\right)
\end{aligned}
$$

where $D_{M_{J} \Omega_{J}}^{J}(\mathbf{a})$ and $D_{\Omega_{L} M_{L}}^{L}(\boldsymbol{\delta})$ are the usual Wigner rotation matrices (ref. 29, p. 154-156) and $\eta= \pm 1$. This expansion can be written as :

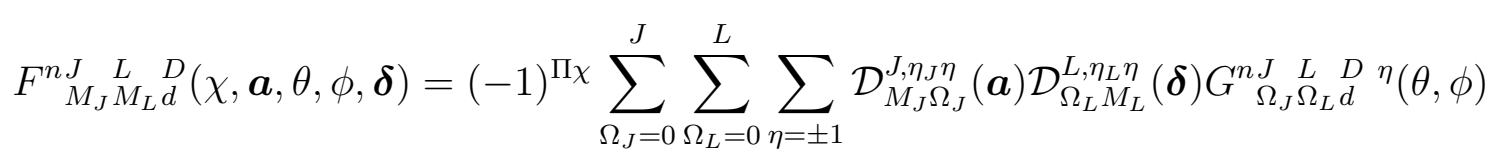

with $\eta_{J}=(-1)^{J}$ and $\eta_{L}=(-1)^{L}$. The $G(\theta, \phi)$ functions, which appear in the expansion of the $F$ functions, are two-angle principal-axes-of-inertia hyperspherical harmonics. In 
the present implementation, they are obtained by expansion on simple symmetry adapted trigonometric primitive basis functions and by diagonalization of the $\hat{\Lambda}^{2}$ operator matrix representation in this basis, as described in ref. [27].

These harmonics can be further symmetrized with respect to permutations of the nuclei. This requires the knowledge of the effects of these permutations on the coordinates of the system. These are given in ref. 20 (eq. 3.37-3.47) for the 6 elements of the permutation group $S_{3}$ of 3 particles. This group contains the identity operation, 3 permutations of 2 particles ( $\hat{P}_{12}, \hat{P}_{13}$ and $\hat{P}_{23}$ for the permutation of particles 1 and 2, 1 and 3,2 and 3, respectively) and 2 cyclic permutations. It has 2 one-dimensional irreducible representations (IRREP) and one two-dimensional one, which are labeled $\Gamma=A_{1}^{(3)}, A_{2}^{(3)}$ and $E^{(3)}$. We add the unusual superscript (3) to these labels to distinguish them from those of the permutation group $S_{4}$ of 4 particles which are : 2 one-dimensional representations $A_{1}^{(4)}$ and $A_{2}^{(4)}, 1$ two-dimensional representation $E^{(4)}$ and 2 three-dimensional ones $T_{1}^{(4)}$ and $T_{2}^{(4)}$. More information on $S_{3}$ and $S_{4}$, and in particular expressions of the matrices of their IRREPs, is given in ref. [30], p. 224-226. $S_{4}$ is the full permutation group of the $\mathrm{Ne}_{4}$ system and must be used to classify its vibrational states. The effets of the permutations on the ROHC are known for the 6 elements of $\mathrm{S}_{4}$ which also belong to $\mathrm{S}_{3}$, but not for its 18 remaining other elements. Hyperspherical harmonics fully symmetrized with respect to $\mathrm{S}_{4}$ are thus not available. Instead, we expand in the present work the $\mathrm{Ne}_{4}$ vibrational states on the harmonics symmetrized with respect to $S_{3}$ and we show how to classify the resulting bound states according to the IRREPs of $S_{4}$.

Using eq. $3.30,3.37-3.47$ of ref. 20 which give the effects of the permutations of $S_{3}$ on the coordinates of the system, we obtain :

$$
\begin{aligned}
& \hat{P}_{12} F_{M_{J} M_{L}}^{{ }^{L}} \underset{d}{D}(\chi, \boldsymbol{a}, \theta, \phi, \boldsymbol{\delta})=(-1)^{n}(-1)^{L} F_{M_{J}-M_{L} d}^{{ }^{D}} \underset{d}{D}(\chi, \boldsymbol{a}, \theta, \phi, \boldsymbol{\delta}) \\
& \hat{P}_{13} F_{M_{J} M_{L}{ }_{L}{ }_{d}}^{D}(\chi, \boldsymbol{a}, \theta, \phi, \boldsymbol{\delta})=(-1)^{n}(-1)^{L} e^{i 4 \pi \frac{M_{L}}{3}} F_{M_{J}-M_{L} d}^{n J}(\chi, \boldsymbol{a}, \theta, \phi, \boldsymbol{\delta}) \\
& \hat{P}_{23} F_{M_{J} M_{L} d}^{L} \underset{d}{D}(\chi, \boldsymbol{a}, \theta, \phi, \boldsymbol{\delta})=(-1)^{n}(-1)^{L} e^{i 2 \pi \frac{M_{L}}{3}} F_{M_{J}-M_{L} d}^{n^{L}}(\chi, \boldsymbol{a}, \theta, \phi, \boldsymbol{\delta})
\end{aligned}
$$

The combinations :

$$
F_{\eta M_{J} M_{L} d}^{n J} \underset{L}{L}(\chi, \boldsymbol{a}, \theta, \phi, \boldsymbol{\delta})=F_{M_{J} M_{L} d}^{n J} \underset{L}{L}(\chi, \boldsymbol{a}, \theta, \phi, \boldsymbol{\delta})+\eta F_{M_{J}}^{n J} \underset{M_{L} d}{L}(\chi, \boldsymbol{a}, \theta, \phi, \boldsymbol{\delta})
$$

are even $\left(\eta=(-1)^{n}(-1)^{L}\right)$ or odd $\left(\eta=(-1)^{n}(-1)^{L+1}\right)$ with respect to $\hat{P}_{12}$. Furthermore, when $M_{L}$ is a multiple of 3 , they are similarly even and odd with respect to $\hat{P}_{13}$ and $\hat{P}_{23}$. 
Thus, the harmonics $F_{\eta M_{J} M_{L}}^{n J} \underset{d}{L}$ belong to $A_{1}^{(3)}$ when $\eta=(-1)^{n}(-1)^{L}$ or to $A_{2}^{(3)}$ when $\eta=$ $(-1)^{n}(-1)^{L+1}$. When $M_{L}$ is not a multiple of 3 , the pair of harmonics $F_{+1 M_{J} M_{L} d}^{J}$ and

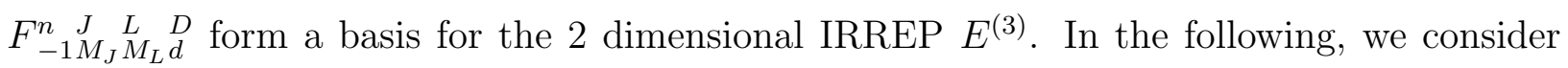
only the harmonics $F_{\eta M_{J} M_{L} d}^{n J} \underset{D}{L}$ with $M_{L}$ multiple of 3 and with $\eta=(-1)^{L}$, consequently, we compute bound states which belong exclusively to $A_{1}^{(3)}$ for even inversion symmetry $(\Pi=0)$ and to $A_{2}^{(3)}$ for odd inversion $(\Pi=1)$.

\section{Bound state computation}

The method used here to obtain 4-body bound states is an extension of the 3-body roworthonormal hyperspherical harmonics expansion method already used to obtain $\operatorname{Ar}_{3}[31]$, $\mathrm{H}_{3}^{+}[32], \mathrm{He}_{3}^{+}[33]$ and $\mathrm{Ar}_{3}^{+}[34]$ rovibrational spectra. The Hamiltonian of the system is obtained by adding the 6 dimensional interaction potential of the system $V(\rho, \theta, \phi, \delta)$ to the kinetic energy operator given by eq. 9. We wish to compute a set of $N_{b}$ eigenstates $\Psi_{k}^{J M_{J} \Pi \Gamma}(\rho, \chi, \boldsymbol{a}, \theta, \phi, \delta)\left(k=1-N_{b}\right)$ of this Hamiltonian with appropriate symmetry properties. These bound states are obtained by expansion on a basis of $N_{s}$ LHSF $\Phi_{i}^{J M_{J} \Pi \Gamma}(\chi, \boldsymbol{a}, \theta, \phi, \boldsymbol{\delta} ; \rho)\left(i=1-N_{s}\right)$, which depend parametrically on $\rho$ and which are defined as eigenstates of a partial fixed- $\rho$ Hamiltonian :

$$
\left(\frac{\hat{\Lambda}^{2}}{2 \mu \rho^{2}}+V(\rho, \theta, \phi, \boldsymbol{\delta})\right) \Phi_{i}^{J M_{J} \Pi \Gamma}(\chi, \boldsymbol{a}, \theta, \phi, \boldsymbol{\delta} ; \rho)=\epsilon_{i}^{J \Pi \Gamma}(\rho) \Phi_{i}^{J M_{J} \Pi \Gamma}(\chi, \boldsymbol{a}, \theta, \phi, \boldsymbol{\delta} ; \rho)
$$

The LHSF potential energy curves $\epsilon_{i}^{J \Pi \Gamma}(\rho)$ are a useful tool to gain insight on the physical properties of the system $[8,9]$ (see section III B below).

We consider a regular hyperradial grid $\rho_{m}$ of $N_{\rho}$ points and the associated $d_{j}(\rho)$ $\left(j=1, N_{\rho}\right)$ Discrete Variable Representation (DVR) functions [35, 36]. These are localized functions obtained from a basis of sine functions such that $: d_{j}\left(\rho_{m}\right) \propto \delta_{j m}\left(\delta_{j m}\right.$ being here the Kronecker symbol). We now form the $N_{\rho} \times N_{s}$ product functions $d_{j}(\rho) \Phi_{i}^{J M \Pi \Gamma}\left(\chi, \boldsymbol{a}, \theta, \phi, \delta ; \rho_{j}\right)$, these are non-direct products since each LHSF is considered at the grid point $\rho_{j}$ associated to the DVR function $d_{j}(\rho)$. These product functions form a basis set on which to expand the bound states :

$$
\Psi_{k}^{J M_{J} \Pi \Gamma}(\rho, \chi, \boldsymbol{a}, \theta, \phi, \boldsymbol{\delta})=\frac{1}{\rho^{4}} \sum_{i, j} a_{i j}^{k J \Pi \Gamma} d_{j}(\rho) \Phi_{i}^{J M_{J} \Pi \Gamma}\left(\chi, \boldsymbol{a}, \theta, \phi, \delta ; \rho_{j}\right)
$$


The coefficients $a_{i j}^{k J \Pi \Gamma}$ are obtained by diagonalizing the matrix representation of the Hamiltonian in this product basis. It is given after renormalization of the Hamiltonian by :

$$
\begin{aligned}
& <d_{j}(\rho) \Phi_{i}^{J M_{J} \Pi \Gamma}\left(\chi, \boldsymbol{a}, \theta, \phi, \boldsymbol{\delta} ; \rho_{j}\right)\left|\rho^{4} \hat{H} \rho^{-4}\right| d_{j^{\prime}}(\rho) \Phi_{i^{\prime}}^{J M_{J} \Pi \Gamma}\left(\chi, \boldsymbol{a}, \theta, \phi, \boldsymbol{\delta} ; \rho_{j^{\prime}}\right)>= \\
& -\frac{\hbar^{2}}{2 \mu}<d_{j}(\rho)\left|\frac{d^{2}}{d \rho^{2}}\right| d_{j^{\prime}}(\rho)>O_{i j, i^{\prime} j^{\prime}}^{J M_{J} \Pi \Gamma}+\left(\frac{6 \hbar^{2}}{\mu \rho_{j}^{2}}+\epsilon_{i}^{J \Pi \Gamma}\left(\rho_{j}\right)\right) \delta_{i i^{\prime}} \delta_{j j^{\prime}}
\end{aligned}
$$

$\delta_{i i^{\prime}}$ and $\delta_{j j^{\prime}}$ are usual Kronecker symbols, $<d_{j}(\rho)\left|\frac{d^{2}}{d \rho^{2}}\right| d_{j^{\prime}}(\rho)>$ is the usual DVR matrix representation of the second derivative operator, as given for instance by appendix A of ref. 37. The overlap matrix elements are given by : $O_{i j, i^{\prime} j^{\prime}}^{J M_{I} \Pi \Gamma}=<\Phi_{i}^{J M_{J} \Pi \Gamma}\left(\chi, \boldsymbol{a}, \theta, \phi, \delta ; \rho_{j}\right) \mid \Phi_{i^{\prime}}^{J M_{J} \Pi \Gamma}\left(\chi, \boldsymbol{a}, \theta, \phi, \boldsymbol{\delta} ; \rho_{j^{\prime}}\right)>$. They replace the usual first derivative couplings which would appear if an adiabatic basis (see eq. 29 below) had been used to expand the wavefunction.

The surface functions $\Phi_{i}^{J M_{J} \Pi \Gamma}(\chi, \boldsymbol{a}, \theta, \phi, \boldsymbol{\delta} ; \rho)$ are obtained from eq. 24 by expansion on a basis of hyperspherical harmonics $F_{\eta M_{J} M_{L}{ }_{L}{ }_{d}}^{D}(\chi, \boldsymbol{a}, \theta, \phi, \delta)$ with quantum numbers selected according to the symmetry $J M_{J} \Pi \Gamma$ considered. Numerical quadratures on a 5 -dimensional grid $N_{\theta} \times N_{\phi} \times N_{\delta_{1}} \times N_{\delta_{2}} \times N_{\delta_{3}}$ are performed for each hyperradial grid point $\rho_{j}$ to obtain the matrix elements of the potential in the harmonic basis. This task represents the most time consuming part of the computation, but it can be performed fully in parallel on different processors for different hyperradii. The matrix elements of the kinetic energy operator are obtained analytically from eq. 10.

In summary, we compute the vibrational states of $\mathrm{Ne}_{4}$ with a basis expansion method which involves 3 successive diagonalization-contraction steps : hyperspherical harmonics are obtained from trigonometric functions, LHSF from harmonics, bound states from LHSF.

\section{Implementation}

We use the same interaction potential $V(\rho, \theta, \phi, \delta)$ as in ref. 12 , this allows for a quantitative comparison between both sets of results. It is a sum of Lennard-Jones pair potentials $v(r)$ which can be expressed as a function of the interatomic distance $r$ by :

$$
v(r)=4 \epsilon\left(\left(\frac{\sigma}{r}\right)^{12}-\left(\frac{\sigma}{r}\right)^{6}\right)
$$

where $\epsilon=24.743267 \mathrm{~cm}^{-1}$ is the 2 body binding potential energy and $\sigma=5.195$ au is a parameter related to the interatomic equilibrium distance $r_{0}$ by $: r_{0}=2^{\frac{1}{6}} \sigma$. In the following, 
all data concerning energy will be given in units of $\epsilon$. The 6 interatomic distances needed in the $\mathrm{Ne}_{4}$ full potential expression are obtained from the Jacobi vectors, which are related to the ROHC by eq. 1. The Neon mass is, as in ref. [12], $19.99244 \mathrm{u}$.

The hyperspherical harmonics belong to an IRREP $\Gamma$ of the subgroup $S_{3}$ of the full 4-body permutation group in our present implementation. We will present in the following results obtained for $\Gamma=A_{1}^{(3)}(\Pi=0)$ and for $\Gamma=A_{2}^{(3)}(\Pi=1)$. The total angular momentum is restricted to $J=0$. An irreducible representation of the group $S_{4}$ is a representation which may be reducible for the subgroup $S_{3}$ and we have the correlations :

$$
A_{1}^{(4)}=A_{1}^{(3)}, \quad A_{2}^{(4)}=A_{2}^{(3)}, \quad T_{1}^{(4)}=A_{2}^{(3)} \oplus E^{(3)}, \quad T_{2}^{(4)}=A_{1}^{(3)} \oplus E^{(3)},
$$

For completeness, we recall the correlation : $E^{(4)}=E^{(3)} . S_{4}$ is isomorphic to $T_{d}$ and the naming conventions of the $T_{d}$ IRREPs can be used for $S_{4} . T_{1}^{(4)}$ and $T_{2}^{(4)}$ are defined such that the character of the permutations of 2 particles $i$ and $j \hat{P}_{i j}(i, j=1-4)$ is -1 for $T_{1}^{(4)}$ and +1 for $T_{2}^{(4)}$. This convention is the one found in most textbooks (see for instance ref. 38, p. 329), albeit opposite to that of ref. 12 following ref. 11. Eq. 28 implies that a $\mathrm{Ne}_{4}$ state computed for $\Gamma=A_{1}^{(3)}\left(\Gamma=A_{2}^{(3)}\right)$ must belong to the $A_{1}^{(4)}$ or $T_{2}^{(4)}\left(A_{2}^{(4)}\right.$ or $T_{1}^{(4)}$ respectively) IRREPs of $S_{4}$. Indeed, the numerical diagonalization process generates automatically eigenstates which belong to IRREPs of the full symmetry group of the operator which is diagonalized, even if these states are expanded on a basis which belongs to a subgroup IRREP.

However, the full group IRREPs to which each eigenstate belongs automatically remains to be found. Usually, it is obtained by repeating the bound state calculations for all the subgroup IRREPs and by analyzing the degeneracies between the resulting bound states. This method was used in ref. 12 to obtain the full group IRREPs which the $\mathrm{Ne}_{4}$ rovibrational states belong to from Jacobi coordinate calculations done for the IRREPs of the $S_{2} \times S_{2}$ subgroup, which contains 4 elements. In the present case, we could have generated the bound states for all $S_{3}$ IRREPs, not only $\Gamma=A_{1}^{(3)}$ which contains the physically allowed states, but also for $\Gamma=A_{2}^{(3)}$ and $\Gamma=E^{(3)}$. We would know from eq. 28 that $A_{1}^{(3)}\left(A_{2}^{(3)}\right)$ bound states which are degenerate with $E^{(3)}$ states belong to the full group IRREP $T_{2}^{(4)}\left(T_{1}^{(4)}\right)$ and that those which are not belong to $A_{1}^{(4)}\left(A_{2}^{(4)}\right.$ respectively). Such an IRREP identification method thus usually requires the computation of extra bound states which are not of direct interest.

A distinctive advantage of the hyperspherical method is that the full group IRREPs 
which each bound state belongs to can be identified without extra calculations. Because the LHSF are obtained by diagonalization of the fixed $\rho$-Hamiltonian (eq. 24) which contains, similarly to the full Hamiltonian, all symmetries of the system, they also belong automatically to IRREPs of the full symmetry group, and not only of the subgroup associated to the hyperspherical harmonics on which they are expanded. Due to the symmetry invariance of the hyperradius $\rho$, a vibrational state belonging to a given (but at this time still unknown) full group IRREP of $S_{4}$ has non zero expansion coefficients $a_{i j}^{k J \Pi \Gamma ~(e q . ~ 25) ~ o n ~ a ~ s u b s e t ~}$ of the LHSF, the one which contains LHSF belonging to the same full group IRREP. By inspection of the expansion coefficient values ( 0 or not), we can separate the set of LHSF resulting from a single, say $A_{1}^{(3)}$, calculation into 2 subsets, one for each full group IRREP, say $A_{1}^{(4)}$ and $T_{2}^{(4)}$. The subset which corresponds to $A_{1}^{(4)}$ is the one which contains the lowest energy LHSF. The full group IRREP to which a given bound state belongs is now known : it is the one of the LHSF subset on which it has non zero expansion coefficients. Neon is a boson with zero nuclear spin and the only physically relevant bound states belong to the full group IRREP $A_{1}^{(4)}$ and are obtained from a single $\Gamma=A_{1}^{(3)}$ calculation. Extra calculations were performed for $\Gamma=A_{2}^{(3)}$ to gain physical insight and to allow for a larger comparison with the results of ref. 12 .

Our convergence goal is to obtain bound state energies within $1 \%$ of the reference data given by [12] and which turn out to be fully converged. This objective was achieved by choosing a truncated harmonic basis set such that $n \leq n_{\max }=50$ and $L \leq L_{\max }=30$ for $\Pi=0, \Gamma=A_{1}^{(3)}$. A basis set of 20289 symmetry adapted harmonics was thus generated on a grid having the following size : $N_{\theta}=51, N_{\phi}=51, N_{\delta_{1}}=N_{\delta_{2}}=N_{\delta_{3}}=37$. The $\theta, \phi, \delta_{2}$ grids are of Gauss-Legendre type while the $\delta_{1}, \delta_{2}$ grids ones are regular Fourier type ones. $N_{S}=100$ surface functions are obtained by diagonalization of the fixed- $\rho$ Hamiltonian in the harmonic basis. A grid of $N_{\rho}=36$ DVR functions is used in the $\rho$ interval $[8.4,15]$ au. Finally, the full Hamiltonian representation in the product basis (eq. 25) is a matrix of size $N_{\rho} \times N_{S}=3600$ which is diagonalized to provide the desired vibrational states and energies. Similar convergence parameters were used for the other symmetry considered $\Pi=1, \Gamma=A_{2}^{(3)}$. 


\section{RESULTS}

\section{A. Energy spectra}

The energy levels resulting from our computations are shown on Table I. Our spectra are in good agreement with those of ref. 12, the relative difference being within $1 \%$. Our results have been obtained using an harmonic basis without any grid optimization. The harmonics which analytically regularize all the singularities of the kinetic energy operator [27] are fully delocalized in the configuration space. When the potential-induced localization of the LHSF in configuration space increases, the convergence rate of their expansion into harmonics decreases. Convergence is thus slower in the large $\rho$ domain and for excited states which extend in this region. Grid optimization would be useful to speed up convergence and limit the CPU time spent in potential quadratures in our present implementation.

The states are labeled on Table I with respect to the IRREPs of $S_{4}$. These labels are obtained by inspection of the weights $a_{i j}^{k J \Pi \Gamma ~ i n ~ t h e ~ e x p a n s i o n s ~ o f ~ t h e ~ v i b r a t i o n a l ~ s t a t e s ~ i n t o ~}$ LHSF (eq. 25), according to the procedure described in subsection IID. Fig. 1 shows the weights integrated over $\rho: \sum_{j}\left|a_{i j}^{k J \Pi \Gamma}\right|^{2}$, for different vibrational states (labelled by $k$ ), as a function of the surface function number $i$. There is a striking oscillatory dependence of the integrated weights as a function of $i$, for a given $k$. This is a symmetry effect : a weight $a_{i j}^{k J \Pi \Gamma}$ is non zero only when the vibrational state $\Psi_{k}^{J M_{J} \Pi \Gamma}$ and the LHSF $\Phi_{i}^{J M_{J} \Pi \Gamma}$ belong to the same full group IRREP. However, as the symmetry character of a given LHSF depends on $\rho$ (there are avoided crossings between LHSF of different $\mathrm{S}_{4}$ symmetries), it is somewhat blurred by integration over $\rho$ and the alternation seen on Fig. 1 for integrated weights is not perfect. It is nevertheless sufficient to assign the vibrational state symmetries. Surface functions and vibrational states can be separated into 2 groups, the group which contains the surface function \# 1 (ground state) is associated to the $A_{1}^{(4)}$ IRREP and the other to $T_{2}^{(4)}$. Alternatively, one may prefer to look at the expansion coefficients at a chosen $\rho$ instead integrating over $\rho$. Because the symmetry character of the surface function at fixed $\rho$ is unambiguous, the alternation equivalent to that of Fig. 1 would be in this case perfect. However, according to the $\rho$ value chosen, the weight of a given LHSF on vibrational states may be very small, and the correlation between bound states and LHSF symmetries may be more difficult to identify. 
The hyperspherical method has 2 other distinctive features which are useful to gain some physical insight. One is to provide surface function energy diagrams $\epsilon_{i}^{J \Pi \Gamma}(\rho)$ (eq. 24) which allow to follow the dynamics as a function of the hyperradius. The other is the separation of the 6 dimensional configuration space into $\mathcal{S}^{\mathcal{K I}}$ and $\mathcal{S}^{\mathcal{K R}}$. We now show how to take advantage of these features to gain a better qualitative understanding of the $\mathrm{Ne}_{4}$ spectra.

\section{B. Potential and surface function energy curves}

Fig. 1 shows that the expansion of a given vibrational state given by eq. 25 converges for a small number of surface functions. It can be seen on the figure that a single surface function is enough to account for $90 \%(80 \%)$ of the ground state of $A_{1}^{(4)}\left(T_{2}^{(4)}\right)$ symmetry. This justifies a posteriori the use of the hyperspherical adiabatic approximation to compute low energy vibrational states of rare gas clusters, as for instance in ref. [9]. Using now the LHSF basis as an adiabatic basis on which to expand the full wavefunction, eq. 25 turns into :

$$
\Psi_{k}^{J M_{J} \Pi \Gamma}(\rho, \chi, \boldsymbol{a}, \theta, \phi, \delta)=\frac{1}{\rho^{4}} \sum_{i} h_{i}^{J M_{J} \Pi \Gamma}(\rho) \Phi_{i}^{J M_{J} \Pi \Gamma}(\chi, \boldsymbol{a}, \theta, \phi, \boldsymbol{\delta} ; \rho)
$$

where the $h_{i}^{J M_{J} \Pi \Gamma}(\rho)$ functions are the coefficients of the expansion in the adiabatic representation. A single product $h_{i}^{J M_{J} \Pi \Gamma}(\rho) \Phi_{i}^{J M_{J} \Pi \Gamma}(\chi, \boldsymbol{a}, \theta, \phi, \boldsymbol{\delta} ; \rho)$ is enough to represent the lowest energy bound state of each symmetry and in this case the hyperradial function $h_{i}^{J M_{J} \Pi \Gamma}(\rho)$ is the one-dimensional bound state supported by the potential energy curve $\epsilon_{i}^{J \Pi \Gamma}(\rho)$ associated to $\Phi_{i}^{J M_{J} \Pi \Gamma}$. Fig. 2 shows a set of such energy curves $\epsilon_{i}^{J \Pi \Gamma}(\rho)$ as a function of the hyperradius, as well as a few low-lying bound state energies.

Interestingly, the expansion of the excited vibrational states converges also with a small number of surface functions. For instance, 2 surfaces functions are sufficient to account for $90 \%(85 \%)$ of the first $A_{1}^{(4)}\left(T_{2}^{(4)}\right)$ excited state at $-2.961 \epsilon(-2.885 \epsilon)$. The two-color segments drawn on Fig. 2 are bounded by pairs of potentials $\epsilon_{i}^{J \Pi \Gamma}(\rho)$ of the same two colors. These potentials are associated to the pair of surface functions involved in the expansions of these vibrational states. Fig. 1 also shows that a few surface functions are sufficient to converge each of the more excited vibrational states. This has the important implication that most of the qualitative characteristics of a given vibrational state are embedded in a few surface functions, those which are involved in the expansion of this vibrational state. 
This is why it is interesting to consider further the properties of the surface function energy diagram shown on Fig. 2.

Asymptotically, the surface functions converge toward the $\mathrm{Ne}+\mathrm{Ne}_{3}$ dissociation limit and their energies are those of the trimer, $-1.71 \epsilon$ (Table II in ref. 39), which corresponds to 0.57 $\epsilon$ of binding energy for each the 3 identical Ne-Ne bonds. The system can also dissociate into two dimers, $\mathrm{Ne}_{2}+\mathrm{Ne}_{2}$. This configuration corresponds however to a higher energy, $-1.13360 \epsilon[40]$, than for the trimer. At the largest $\rho$ value shown on Fig. $2, \rho=14.5$ $\mathrm{au}$, the energy of the lowest surface function is nearly $-2.2 \epsilon$, which is significantly smaller than the asymptote $-1.71 \epsilon$, the difference being related to the attractive nature of the long range part $-r^{-6}$ of the Lennard-Jones potential. The range of $\rho$ shown on Fig. 2 can be separated in 3 domains defined by : $P^{S F}: \rho \lesssim 11, R^{S F}: 11 \lesssim \rho \lesssim 13, T^{S F}: 13 \lesssim \rho$ (in au). The superscript $S F$ refers to the fact that these domains are defined with respect to the characteristics of the Surface Function energy curves $\epsilon_{i}$. Similar domains were defined on the ground surface function energy curve computed in ref. 9 and called "plateau-like regions". The definition of these domains can be extended for higher energy surface function curves, but their boundaries must be shifted to increasingly larger $\rho$.

In the $P^{S F}$ domain, $\Pi=0$ and $\Pi=1$ surface function energies are degenerate by pairs. The potential curves have minima, near $\rho=9.6$ au for the ground state, and near increasingly larger $\rho$ for excited states. States of the same parity $\Pi$ are non degenerate in this domain. On the contrary, in the $R^{S F}$ domain, the $\Pi=0-\Pi=1$ degeneracy is not present, but several states of the same parity are doubly degenerate. In this domain, energy curves have no minima but are convex. In the $T^{S F}$ domain, the energy curves are also convex and some are triply degenerate.

The $P, R$ and $T$ letters are chosen by reference to the Pyramid, Rhombus and Trigonal geometries, as now discussed. These geometries are depicted schematically on Fig. 3. This figure also shows the potential energy minimum curve. At fixed $\rho$, minimization of the potential is performed over the 5 angles $\theta, \phi, \delta$. Minima and curvature effects similar to those associated to $\epsilon_{i}$ can be observed on this minimum potential energy curve, but the bounds of the corresponding domains are shifted in $\rho$. We now have : $P^{\text {min }}: \rho \lesssim 10$, $R^{\text {min }}: 10 \lesssim \rho \lesssim 12, T^{\text {min }}: 12 \lesssim \rho$ (in au). In each domain, the potential minimum corresponds to a different geometry. $P^{\min }$ is the domain where the potential minimum corresponds to a tetrahedron (a triangular Pyramid) geometry. Its 6 edges are formed from 
similar $\mathrm{Ne}-\mathrm{Ne}$ bonds and its 4 faces from similar equilateral $\mathrm{Ne}_{3}$ triangles. The potential is minimum when the tetrahedron edge length is the $\mathrm{Ne}-\mathrm{Ne}$ equilibrium distance $r_{0}$, then $\rho=\sqrt{3} \sigma=8.998$ au $\in P^{m i n}$, and the potential is $-6 \epsilon$ (see Fig. 3). The size of the tetraedron then increases with $\rho$, the 6 equivalent $\mathrm{Ne}-\mathrm{Ne}$ bonds simultaneously weaken gradually and the minimum potential increases. Near $\rho \approx 10 \mathrm{au}$, it becomes energetically more advantageous to break one of these 6 bonds to continue increasing global size at minimum energy cost. The minimum energy structure thus shifts abruptly from a Pyramid to a Rhombus. For instance, by breaking the bond between atoms 1 and 2, the new minimum energy structure becomes a rhombus which we label $1-34-2$, which consists of the 2 equilateral triangles (134) and (234) joined by a common side formed by the bond between atoms 3 and 4 . The angles associated to vertices 1 and 2 are : $\alpha=\pi / 3$. There are of course 6 equivalent Rhombus listed on Table II which can be formed from the Pyramid. 5 bonds provide the cohesion of the rhombus, its potential energy is minimum and is equal to nearly $-5 \epsilon$ when its side length is $r_{0}$, which occurs when $\rho=2 \sigma=10.390$ au $\in R^{\text {min }}$ (see Fig. 3). As the hyperradius increases further, the rhombus size increases, its energy also, but this increase is minimized by a slight distortion which consists in a decrease from $\pi / 3$ of the vertex angle $\alpha$ (see Fig. 3). At some point, however, a Trigonal structure becomes energetically more favorable : $3 \mathrm{Ne}$ atoms form an equilateral triangle from 3 identical bonds, a fourth bond is formed between one of these 3 atoms (say atom 3) and the 4th one, such that the (34) bond bisects bonds (13) and (23). We label this structure 4-3-12. There are 12 such equivalent trigonal structures (see Table III). The energy of these structures is nearly $-4 \epsilon$ when the common bond length is $r_{0}$, which occurs when $: \rho=\sqrt{4+\sqrt{3}} \sigma=12.438$ au $\in T^{\text {min }}$ (see Fig. 3). At some larger $\rho$, a structure more stable than this symmetric trigonal one can obtained by increasing the (34) bond length, but leaving the other 3 bonds unchanged, leading eventually to the asymptotic structure $\mathrm{Ne}+\mathrm{Ne}_{3}$.

The abrupt transitions in the geometries of these constrainted fixed- $\rho$ minimum potential energy structures as the hyperradius increases define 3 domains, $P^{\text {min }}, R^{\text {min }}$ and $T^{\text {min }}$, which have different bounds from those, $P^{S F}, R^{S F}$ and $T^{S F}$, observed on the surface function energy curves. We now analyze the surface functions themselves to understand how they reflect these structural changes as a function of $\rho$. This will allow us to understand why the $\rho$ domain bounds are different for the surface function energies and for the minimum potential energy. 


\section{Surface function analysis}

Analyzing the LHSF themselves is obviously difficult because they belong to the 5dimensional configuration space spanned by the 5 angles $\left(\theta, \phi, \delta_{1}, \delta_{2}, \delta_{3}\right)$. We can take advantage however of the separation of this space into the $\mathcal{S}^{\mathcal{K I}}$ and $\mathcal{S}^{\mathcal{K} \mathcal{R}}$ subspaces.

\section{In $\mathcal{S}^{\mathcal{K} \mathcal{I}}$}

$\mathcal{S}^{\mathcal{K I}}$ contains $\theta$ and $\phi$ and its structure is depicted in ref. [15], Fig. 1. It contains a point $\mathrm{P}$ given by $\theta=\arcsin \sqrt{\frac{2}{3}} \approx 55^{\circ}, \phi=\frac{\pi}{4}$ which corresponds to the same tetrahedral configuration (symmetric top, Pyramid) for all $\delta_{1}, \delta_{2}, \delta_{3}$ values. The point $\theta=0$ (arbitrary $\phi)$ corresponds to the collinear configurations, the point $\mathrm{S}(\theta=\pi / 4, \phi=0)$ to the coplanar symmetric top configurations, different configurations of each type corresponding to different $\delta_{1}, \delta_{2}, \delta_{3}$, i.e. different points in $\mathcal{S}^{\mathcal{K R}}$. One example of such coplanar symmetric top is the square formed by 4 Neon atoms, another one is the equilateral triangle formed by 3 of them, with the 4 th one placed at the center of mass of the triangle. The segment which connects $\mathrm{P}$ to $\mathrm{S}$ and defined by : $\theta=\arcsin \frac{1}{\sqrt{1+\cos ^{2} \phi}}$ contains all oblate symmetric top configurations, like the triangular pyramids derived from the tetrahedron by approaching one apex Ne atom perpendicularly to its equilateral triangular base.

Fig. 4 shows ground and excited LHSF in $\mathcal{S}^{\mathcal{K I}}$ for $\rho=9.6$ au. These functions are all localized in $\mathcal{S}^{\mathcal{K I}}$ near the $P$ point (tetrahedron), as expected since $\rho=9.6$ au $\in P^{S F}$ (and incidentally also $\left.\in P^{\min }\right)$. The plots are performed somewhat arbitrarily for $: \delta_{1}=\delta_{2}=$ $\delta_{3}=\pi / 2$ since the wavefunction value is strictly independent of $\delta_{1}, \delta_{2}, \delta_{3}$ at the $P$ point, and weakly dependent in its vicinity. The localization of the wavefunctions near $P$ results from the presence of a potential barrier near coplanarity $(\phi=0)$. This barrier prevents the system from changing chirality during its vibration as it would if it could pass through the coplanar geometry. As a result, $\Pi=0$ wavefunctions are degenerate with those for $\Pi=1$ and the plots for $\Pi=1$ would be identical to those of Fig. 4 . Fig. 4 also shows the nodal lines associated to vibrational excitations. The $P S$ line is an antinodal (nodal) line for the $A_{1}^{(4)}\left(T_{2}^{(4)}\right)$ symmetry. A motion along the $P S$ line corresponds to vibrations of the Ne atoms which preserve the symmetric top character. The nodal lines which are approximately perpendicular to the $P S$ line in $\mathcal{S}^{\mathcal{K I}}$ (bottom row of Fig. 4 for both permutation symmetries) 
are other traces of vibrational excitation.

Fig. 5 and 6 show the changes of the ground LHSF characteristics as a function of $\rho$. The 3 $\rho$ values are selected such that $: \rho=10.6$ au $\in P^{S F}, \rho=11.6$ au $\in R^{S F}$ and $\rho=13.6$ au $\in T^{S F}$, so that the gradual change in $\mathcal{S}^{\mathcal{K I}}$ (left column) from pyramid to rhombus and trigonal configurations is visible on the figures. At $\rho=10.6$ au (Fig. 5 top left), the wavefunction is still mainly localized in the vicinity of $P$, as for $\rho=9.6$ au (Fig. 4), however a transition toward coplanarity starts to appear by an extension of the wavefunction with significant amplitude in this direction $(\phi=0)$. At $\rho=11.6$ au (Fig. 5 bottom left), the surface function already has a significant amplitude in the coplanarity region and as a result, the degeneracy between $\Pi=0$ and $\Pi=1$ pair of states disappears.

It is interesting to notice that the wavefunction at $\rho=10.6$ au is not localized in the minimum potential region $(\phi=0$ line $=$ coplanar configurations $)$, but in the low kinetic energy region. In fact, the wavefunction remains localized far from coplanarity until the potential near $P$ becomes larger than the surface function energy. This is shown on Fig. 3 : the transition between $P^{S F}$ and $R^{S F}$ occurs near $\rho=11$ au when the potential at $P$ (dashed line) becomes larger than the lowest surface function energy. The $P$ point region then becomes energetically unreachable for the wavefunction, which is pushed toward the coplanar region by the potential as $\rho$ increases. The fact that $P^{S F}$ extends up to 11 . au, as compared to $P^{\text {min }}$ which extends up to 10 . au only, is thus related to the fact that the wavefunction localization is governed by the turning point region, rather than by the minimum potential region. A similar phenomenon occurs for the transition from $R^{S F}$ to $T^{S F}$, between rhombus and trigonal configurations. The transition occurs when the potential associated to the rhombus configuration (dashed line, $\alpha=60^{\circ}$ ) crosses the lowest surface function energy curves near $\rho=13$. au (Fig. 3) so that the rhombus region becomes energetically unreachable, and not near $\rho=12$. au (the boundary between $R^{\text {min }}$ and $T^{\text {min }}$ ) when the potential minimum switches from rhombus to trigonal geometries. Because $\rho=$ $11.6 \mathrm{au} \in R^{S F}$ and simultaneously $\in R^{\text {min }}$, the corresponding wavefunction (Fig. 5, left column, bottom row) is localized near coplanarity, which is also the region of minimum potential. A similar observation can be made in the trigonal region for the wavefunction at $\rho=13.6$ au $\in T^{S F}$ and simultaneously $\in T^{\text {min }}$ (Fig. 6, left column, bottom row). In summary, we can say that the LHSF localization is dynamically (turning points driven) rather than statically (potential driven) controlled. 


\section{In $\mathcal{S}^{\mathcal{K} \mathcal{R}}$}

Plots of the LHSF in $\mathcal{S}^{\mathcal{K} \mathcal{R}}$ provides complementary useful information (Fig. 5, central and right columns, Fig. 6, right and Fig. 7, right). Coplanar configurations of special interest are the 6 rhombus and 12 trigonal ones already mentioned, their coordinates have been obtained with the method described in appendix A and are listed in Tables II and III. The plots are presented in specific planes of $\mathcal{S}^{\mathcal{K} R}$ selected such that they contain several of these important configurations and such that the surface function amplitude is significant. The ground surface function is shown on Fig. 5 and 6 . The most striking feature is the localization of the functions near the 6 points $R_{1}-R_{6}$ at $\rho=11.6$ au and near the 12 points $T_{1}-T_{12}$ at $\rho=13.6$ au. This localization was expected since $\rho=11.6$ au $\in R^{S F}$ and $\rho=13.6$ au $\in T^{S F}$. It is important to realize that this localization is the reason why surface functions are degenerate in the $R^{S F}$ and $T^{S F}$ domains (Fig. 2 and 3). Indeed, let us call $\Phi_{R_{m}}(m=1-6)\left(\Phi_{T_{m}}(m=1-12)\right)$ wavefunctions which are localized exclusively in the vicinity one of these 6 (12) points. The symmetrized LHSF shown on the figures are linear combinations of these. Indeed, the $6 \Phi_{R_{m}}$ are degenerate in $R^{S F}$, the $12 \Phi_{T_{m}}$ in $T^{S F}$, and each of these is, like the LHSF $\Phi^{J M_{J} \Pi \Gamma}$, an eigenfunction of the fixed $\rho$-Hamiltonian, but without the appropriate permutation symmetry properties. We show in appendix B how to use group representation theory to form the appropriate linear combinations of these localized functions which restore the LHSF with the expected permutation symmetries. We also show that this $\mathcal{S}^{\mathcal{K} \mathcal{R}}$ localization explains the appearance of degenerate pairs (triplets) in

the $R^{S F}\left(T^{S F}\right)$ domain. As surface function localization decreases as $\rho$ decreases (compare for instance the surface functions in $\mathcal{S}^{\mathcal{K} \mathcal{R}}$ at $\rho=10.6$ au, Fig. 5 top row, vs $\rho=11.6$ au, Fig. 5 bottom row), $\mathcal{S}^{\mathcal{K} R}$-induced degeneracies disappear in the $P^{S F}$ domain (but $\mathcal{S}^{\mathcal{K I}}$-induced ones appear, see section III C 1). Fig. 7 also shows excited surface functions in $\mathcal{S}^{\mathcal{K} R}$. Surface functions at the same $\rho$ may have different energies but similar shapes in $\mathcal{S}^{\mathcal{K}}$, as if they had similar excitation in this subspace. For instance, the surface function $\# 3$ at $\rho=11.6$ au (Fig. 7, top-right) is similar in $\mathcal{S}^{\mathcal{K} \mathcal{R}}$ to function \# 1 (Fig. 5 , bottom right), as well as to functions \#2 and \#4 (not shown). In this case, excitation is visible in $\mathcal{S}^{\mathcal{K I}}$ (similar to that shown on Fig. 4 at $\rho=9.6 \mathrm{au}$ ). In other cases, excitation also appears in $\mathcal{S}^{\mathcal{K} \mathcal{R}}$, as shown on Fig. 7 (bottom right) for state \# 5 which has a $\delta_{1}$ excitation. We show in appendix B that the state of excitation has impact on the symmetry properties and on the degeneracies of the 
surface functions, for instance states \#1-4 without excitation in $\mathcal{S}^{\mathcal{K} \mathcal{R}}$ are doubly degenerate in $R^{S F}$ whereas state $\# 5$ with $\delta_{1}$-excitation is non degenerate.

\section{Bound state analysis}

As the bound states are linear combinations of the LHSF (eq. 29) which involve a few termes only (Fig. 1), the properties of the bound states are directly related to those of the surface functions. Moreover, at least for the low energy states, the coefficients of this expansion are localized in $\rho$ so that the properties of the bound state reflect those of the surface functions in a limited number of domains. For instance, bound state \# 1 (for both parities) is localized in the $P^{S F}$ domain (see Fig. 2). As a result, the bound state wavefunction in localized near $\mathrm{P}$ in $\mathcal{S}^{\mathcal{K} \mathcal{I}}$ (see Fig. 4) and is associated to the tetrahedral geometry. States from \#2 to \#10 have energies below $-2.55 \epsilon(-2.50 \epsilon)$ for $\Pi=0(\Pi=1)$, see Table I. These states are localized in $P^{S F}+R^{S F}$, the corresponding motion is one of a pyramid experiencing large amplitude rotations around its edges (say the one connecting atoms 1 and 2 ) so that the equilateral triangles (123) and (124) can explore the coplanar geometries and in particular the rhombus one $R_{1}=3-12-4$. The motion extends symmetrically $(\Pi=0)$ or antisymmetrically $(\Pi=1)$ on the other side of the rhombus plane and the tetraedron geometry with opposite chirality can be explored. This vibrational motion between tetrahedron and rhombus configurations corresponds to an oscillation motion in $\rho$ between $P^{S F}$ and $R^{S F}$ described by the $h_{i}^{J M_{J} \Pi \Gamma}(\rho)$ functions (eq. 29). The same rotational motion can occur around the 6 edges of the tetraedron, which corresponds to exploring the 6 rhombus configurations $R_{1}-R_{6}$ (Table II) with identical, opposite or different amplitudes, according to the possible symmetries of the wavefunction in $\mathcal{S}^{\mathcal{K} R}$. As long as the bound states are in $P^{S F}$, they do not explore the coplanarity region and tunneling splitting is very small. This splitting increases however for higher energy bound states which extend in the $R^{S F}$ region which corresponds to coplanarity. At even higher energies (state \#11 and above) the motion can be even more delocalized in $P^{S F}+R^{S F}+T^{S F}$, which corresponds to an even larger amplitude motion in $\rho$. In this case, an in-plane motion, where the rhombus is distorted into the trigonal geometry, adds to the out of plane motion between tetrahedron and rhombus configurations. Tunneling splitting remains large.

A splitting of the $\mathrm{Ne}_{4}$ vibrational spectrum in 3 regions is proposed in ref. [12]. It is based 
on the analysis of the increase of the tunneling splittings and of the density of vibrational states (DOS) with energy. The low energy region contains a few low lying states (states \# 1 of both parities on Table I) which are "highly localized". The intermediate energy region corresponding approximately to the interval $[-3.4 \epsilon,-2.6 \epsilon]$ (from states \# 2 to \#8 on Table I) is associated to an intermediate DOS of "fluxional" states with intermediate tunneling splittings. The high energy region (above $-2.6 \epsilon$ ) corresponds to a high DOS of completely delocalized vibrational states. The present hyperspherical analysis provides a complementary view on this spectrum splitting. From inspection of Fig. 2 and 3 in relation to the energy bounds of these 3 regions, it is clear that the low energy region corresponds to the states which extend in $P^{S F}$, the intermediate energy region to the states which extend in $P^{S F}+R^{S F}$ and finally the high energy region to the states which extend in $P^{S F}+R^{S F}+T^{S F}$. The increase with energy of the vibrational DOS mentioned in ref. [12] is thus related to an increase of the configuration space volume occupied by vibrational states of increasing energy. The increase of the tunneling splitting is related to the accessibility of the coplanar region in $R^{S F}$ and $T^{S F}$.

\section{SUMMARY AND CONCLUSIONS}

We have presented in the present paper the first implementation of the row-orthonormal hyperspherical coordinate formalism on a tetratomic system. The method provides not only spectra with reasonable accuracy, but also physical insight into the vibrational dynamics of the system. In particular, we have related features of the spectra, like permutation and inversion symmetry degeneracies, inversion tunneling splittings and densities of states, to the way the wavefunction is localized in configuration space. There is however an obvious shortcoming in our approach which should be remedied in the future. We have used the subgroup $\mathrm{S}_{3}$ of the full permutation group $\mathrm{S}_{4}$ of the system. As a result, our computed spectra are superpositions of spectra associated to different irreducible representations of $\mathrm{S}_{4}$. Although we have shown how to disentangle simply mixed symmetries within the hyperspherical formalism, performing separate computations for the different irreducible representations of $\mathrm{S}_{4}$ would reduce the computational cost of the calculations. This requires first to identify the effect of all the 24 elements of $\mathrm{S}_{4}$ on the coordinates of the system and to symmetrize accordingly the Wigner rotation matrices on which the hyperspherical harmonics are expanded. 
This is a natural continuation of the present work.

\section{Data availability}

The data and computer programs that support the findings of this study are available from the author upon reasonable request.

\section{Acknowledgments}

We thank prof. Christoph Meier for stimulating discussions. 


\section{Appendix A: The hyperspherical coordinates for rhombus and trigonal geometries}

Finding the hyperspherical coordinates for a given geometry of the system requires solving non linear equations to obtain them from the mass-scaled Jacobi coordinates in the body frame : $\boldsymbol{\rho}^{b f}=\rho \mathbf{N}(\theta, \phi) \tilde{\mathbf{R}}\left(\delta_{1}, \delta_{2}, \delta_{3}\right)$. Solving these equations is in general a difficult task but for specific geometries simple solutions exist. For the rhombus geometries, we first consider the configurations where atoms 1 and 2 are located at opposite vertices, namely $R_{1}: 3-12-4$ and $R_{6}:$ 1-34-2. In these cases, the Jacobi vectors $\mathbf{r}^{(2)}$ and $\mathbf{r}^{(3)}$ are collinear and both perpendicular to $\mathbf{r}^{(1)}$, this special geometries make the extraction of the hyperspherical coordinates easier. The hyperspherical coordinates for all other rhombus configurations could be obtained from a single one, say $R_{1}$, if the effects on coordinates of all atom permutations belonging to $\mathrm{S}_{4}$ were known. Unfortunately, simple expressions are available only for the transformations which belong to the subgroup $\mathrm{S}_{3}$ of $\mathrm{S}_{4}$. The permutations of $\mathrm{S}_{3}$ must be applied on 2 configurations, say $R_{1}$ and $R_{6}$, to cover all 6 configurations. The hyperspherical coordinates for $R_{2}$ are obtained from those for $R_{1}$ by applying the coordinate transformation associated to the permutation of atoms 1 and 3 and given by eq. 3.40 of ref. [20]. Similarly, the hyperspherical coordinates for $R_{3}$ are obtained from those for $R_{1}$ by applying the coordinate transformation associated to the permutation of atoms 2 and 3 and given by eq. 3.38 of ref. [20]. A similar procedure can be used to obtain the coordinates associated to $R_{4}$ and $R_{5}$ starting from $R_{6}$. The results for the 6 rhombus configurations are collected on Table II.

A similar procedure can be used to obtain the hyperspherical coordinates of the 12 trigonal configurations. $T_{1}, T_{4}$ and $T_{12}$ are special configurations for which the hyperspherical coordinates can be obtained easily from the Jacobi vectors. $T_{2}$ and $T_{3}$ coordinates are obtained from $T_{1}$ by permutations, $T_{5}$ and $T_{6}$ from $T_{4}$; the 5 configurations $T_{7}$ to $T_{11}$ are similarly obtained from $T_{12}$. The results for the 12 trigonal configurations are collected on Table III. 


\section{Appendix B: Group theory, degeneracy and symmetry of surface functions}

We discuss the degeneracies of the surface functions observed on Fig. 2 and 3 : double degeneracies appear in the $R^{S F}$ domain (rhombus) and triple degeneracies in the $T^{S F}$ domain (trigonal). We relate these degeneracies to the symmetry properties of the surface functions.

For instance, in the $R^{S F}$ domain near $11.6 \mathrm{au}$, the surface function with excitation number \# $1\left(A_{1}^{(4)}\right.$ symmetry) is almost degenerate with surface function \# $2\left(T_{2}^{(4)}\right)$. Similarly, the surface function \# $3\left(A_{1}^{(4)}\right)$ is almost degenerate with surface function \# $4\left(T_{2}^{(4)}\right)$. On the other hand, surface function \#5 $\left(T_{2}^{(4)}\right)$ is non degenerate. See Fig. 3 which shows the surface function numbers. The surface functions \# 1 and \# $3\left(A_{1}^{(4)}\right)$ have similar amplitudes in the vicinity of all rhombus points $R_{1}-R_{6}$ (the surface function $\# 1$ is shown on Fig. 5, central and right columns, for $\rho=11.6 \mathrm{au}$ ), whereas the amplitude near $R_{4}-R_{6}$ is opposite to the one near $R_{1}-R_{3}$ for surface functions \# 2 and \# $4\left(T_{2}^{(4)}\right.$ symmetry, not shown). The non degenerate surface function \# 5 has a $\delta_{1}$ excitation (Fig. 7 , bottom right).

In the $T^{S F}$ domain near $\rho=13.6$ au, the 3 lowest surface functions are degenerate (see Fig. 3) and among them, 2 have the $T_{2}^{(4)}$ symmetry and the 3 rd one is $A_{1}^{(4)}$. The surface function energy \# 4 has $T_{2}^{(4)}$ symmetry and is non degenerate.

We now show how these degeneracy and symmetry properties can be accounted for using group theory. We take advantage of the fact that the surface functions are linear combinations of disconnected wavefunctions localized in the $\left(\delta_{1}, \delta_{2}, \delta_{3}\right)$ subspace near the rhombus $R_{1}-R_{6}$ or trigonal $T_{1}-T_{12}$ points (see for example Fig. 5, central and right columns, Fig. 6, right column, as well as Fig. 7 , right column). We call $\Phi_{R_{m}}(m=1-6)$ and $\Phi_{T_{m}}$ $(m=1-12)$ these localized wavefunctions. They are degenerate solutions of the fixed- $\rho$ Hamiltonian, but on the contrary of the surface functions $\Phi_{i}^{J M_{J} \Pi \Gamma}$ which are also solutions of eq. 24 , they are not basis functions of an IRREP of the permutation group $S_{4}$. However, a 6-dimensional (12-dimensional) reducible representation of this group can be obtained from the $\Phi_{R_{i}}(i=1-6)$ basis functions $\left(\Phi_{T_{i}}(i=1-12)\right)$ which we call $\boldsymbol{\Gamma}^{R}\left(\boldsymbol{\Gamma}^{T}\right)$ and which can be decomposed into the IRREPs of $S_{4}$ using the usual projection techniques ([38], p. 29-43).

We first assume that the $\Phi_{R_{m}}$ functions are nodeless in the $\left(\delta_{1}, \delta_{2}, \delta_{3}\right)$ subspace. This is the case for instance for the functions \# 1 to \# 4 at $\rho=11.6$ au (function \# 1 is shown on Fig. 5 and \#3 on Fig. 7 ). In this case, applying $\hat{P}_{i j}$ on $\Phi_{R_{m}}$ changes it into another $\Phi_{R_{m^{\prime}}}$ 
without sign change of the amplitude. Using Table II, we obtain for instance:

$$
\hat{P}_{12} \Phi_{R_{1}}=\Phi_{R_{1}}, \hat{P}_{23} \Phi_{R_{1}}=\Phi_{R_{3}}, \hat{P}_{34} \Phi_{R_{1}}=\Phi_{R_{1}}
$$

and similarly for the other $\Phi_{R_{m}}$ functions. The effect of the other permutations of $\mathrm{S}_{4}$ can be obtained by combination of these 3 generators of $\mathrm{S}_{4}$. We thus obtain the 6 dimensional reducible representations of the 3 generators of $S_{4}$ in the $\Phi_{R_{m}}$ basis :

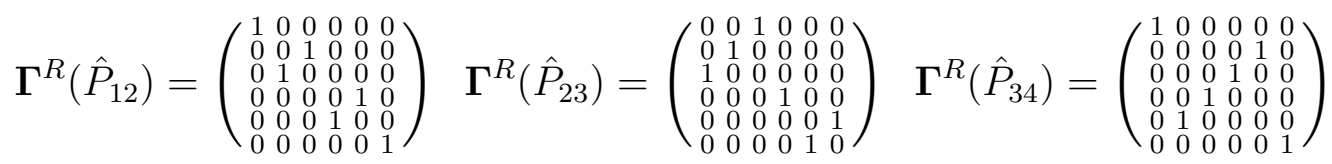

The IRREPs of $S_{4}$, the 1-dimensional ones $A_{1}^{(4)}$ and $A_{2}^{(4)}$, the 2-dimensional one $E^{(4)}$ and the 3-dimensional ones $T_{1}^{(4)}$ and $T_{2}^{(4)}$, are well known (ref. 30, p. 224-226). Using these and the orthogonality relation for characters (eq. 3.20, p. 30 in ref. 38), we obtain the following decomposition of $\Gamma^{R}: \Gamma^{R}=A_{1}^{(4)} \oplus E^{(4)} \oplus T_{2}^{(4)}$. Because of the known correlation between $S_{3}$ and $S_{4}$ IRREPs, we know that only 1 of the 3 basis functions belonging to $T_{2}^{(4)}$, as well as the single one of $A_{1}^{(4)}$, can also be basis functions for $A_{1}^{(3)}$. These $\Phi_{A_{1}^{(4)}}$ and $\Phi_{T_{2}^{(4)}}$ functions form the degenerate pairs (functions \#1-2 and \#3-4) observed on Fig. 3 in $R^{S F}$. Using the projector operator technique (eq. 3.36, p. 41, in ref. 38), these functions are expressed in terms of the basis function of the reducible representation as : $\Phi_{A_{1}^{(4)}}=\frac{1}{\sqrt{6}} \sum_{m=1-6} \Phi_{R_{m}}$ and $\Phi_{T_{2}^{(4)}}=\frac{1}{\sqrt{6}}\left(\sum_{m=1-3} \Phi_{R_{m}}-\sum_{m=4-6} \Phi_{R_{m}}\right)$. This explains why the amplitude of the $A_{1}^{(4)}$ surface function of a degenerate pair in $R^{S F}$ is the same for all rhombus points $R_{1}-R_{6}$, while the amplitude near $R_{4}-R_{6}$ is opposite to the one near $R_{1}-R_{3}$ for the other surface function of $T_{2}^{(4)}$ symmetry.

We now consider a function with a $\delta_{1}$ excitation, like the state \# 5 shown on Fig. 7 $(\rho=11.6 \mathrm{au})$. Although it is like states \# 2 and 4 a $T_{2}^{(4)}$ state, it has significant and similar amplitudes only in the vicinity of $R_{4}-R_{6}$ and negligible ones near $R_{1}-R_{3}$. Also, there is no corresponding $A_{1}^{(4)}$ state to form a degenerate pair with. These observations can also be accounted for by group theory considerations. This surface function with $\delta_{1}$ excitation is obtained by linear combination of new $\Phi_{R_{m}}^{\prime}(m=1-6)$ basis functions, which are localized similarly to the $\Phi_{R_{m}}$ ones but differ from them by a $\delta_{1}$ excitation similar to that of the surface function. A function $\Phi_{R_{m}}^{\prime}$ associated to the rhombus $m=i-j k-l$ (see Table II) behave like $\Phi_{R_{m}}$ under the action of $\hat{P}_{12}, \hat{P}_{23}$ and $\hat{P}_{34}$, except for the central ones : $\hat{P}_{12} \Phi_{R_{1}}^{\prime}=-\Phi_{R_{1}}^{\prime} \ldots$ We thus obtain the following new 6 dimensional reducible representations 
$\Gamma^{R^{\prime}}$ of the 3 generators of $\mathrm{S}_{4}$ in the $\Phi_{R_{m}}^{\prime}$ basis :

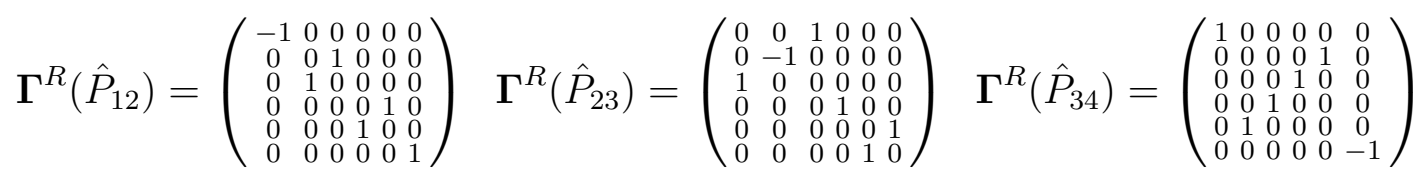

We now have : $\Gamma^{R^{\prime}}=T_{2}^{(4)} \oplus T_{1}^{(4)}$. None of the $T_{1}^{(4)}$ basis functions and a single $T_{2}^{(4)}$ one can belong to $A_{1}^{(3)}$, this explains why state $\# 5$ in $R^{S F}$ belongs to $T_{2}^{(4)}$ and is non degenerate.

In the $T^{S F}$ domain, the surface functions are localized in the vicinity of the trigonal points $T_{1}-T_{12}$ (see for example Fig. 6, right column) and they can be obtained as linear combinations of the localized functions $\Phi_{T_{m}}(m=1-12)$. These form a 12-dimensional representation $\Gamma^{T}$ of $S_{4}$ which can be reduced into its IRREPs using the same techniques as in the $R^{S F}$ domain. We obtain : $\Gamma^{T}=A_{1}^{(4)} \oplus E^{(4)} \oplus 2 T_{2}^{(4)} \oplus T_{1}^{(4)}$. As only $A_{1}^{(4)}$ and $T_{2}^{(4)}$ can have basis functions belonging to $A_{1}^{(3)}$, we expect a triple degeneracy for the lowest energy states in the $T^{S F}$, as observed on Fig. 3, which consists of a single $A_{1}^{(4)}$ state and a pair of $T_{2}^{(4)}$ states. State \#4 has a $\delta_{1}$ excitation in the $T^{S F}$ domain. We use localized functions $\Phi_{T_{m}}^{\prime}$ $(m=1-12)$ with appropriate excitations such that they are unchanged by permutation of 2 atoms except the pair of end atoms $k l$ in the trigonal structure $i-j-k l$ (Table III) : $\hat{P}_{12} \Phi_{T_{1}}^{\prime}=-\Phi_{T_{1}}^{\prime} \ldots$ The 12 dimensional reducible representation $\Gamma^{T^{\prime}}$ of $S_{4}$ in the $\Phi_{T_{m}}^{\prime}$ is likewise reduced into its IRREPs : $\Gamma^{T^{\prime}}=A_{2}^{(4)} \oplus E^{(4)} \oplus T_{2}^{(4)} \oplus 2 T_{1}^{(4)}$. A single basis function, the one associated to $T_{2}^{(4)}$, can also belong to $A_{1}^{(3)}$. This is the reason why state \# 4 belongs to $T_{2}^{(4)}$ and is non degenerate in the $T^{S F}$ domain.

Notice finally that the degeneracies observed in the $R^{S F}$ and $T^{S F}$ domains result from surface function localizations in $\mathcal{S}^{\mathcal{K} R}$. In the $P^{S F}$ domain, surface functions are delocalized in $\mathcal{S}^{\mathcal{K R}}$ and these degeneracies disappear. Instead, new degeneracies between $\Pi=0$ and $\Pi=1$ surface functions appear in this domain and they are induced by localization of the surface functions near $\mathrm{P}$ in $\mathcal{S}^{\mathcal{K I}}$ (see section III C 1). 
[1] S. Althorpe and D. Clary, Annu. Rev. Phys. Chem. 54, 493 (2003).

[2] B. K. Kendrick, J. Chem. Phys. 148, 044116 (2018).

[3] C. H. Greene, P. Giannakeas, and J. Pérez-Ríos, Rev. Modern Phys. 89, 035006 (2017).

[4] L. Marcucci, J. Dohet-Eraly, L. Girlanda, A. Gnech, A. Kievsky, and M. Viviani, Frontiers in Physics 8, 69 (2020).

[5] M. Márquez-Mijares, O. Roncero, P. Villarreal, and T. González-Lezana, Int. Rev. Phys. Chem. 37, 329 (2018).

[6] S. T. Rittenhouse, J. von Stecher, J. P. DIncao, N. P. Mehta, and C. H. Greene, J. Phys. B : At. Mol. Opt. Phys. 44, 172001 (2011).

[7] C. Greene, Physics Today 63, 40 (2010).

[8] D. Blume and C. H. Greene, J. Chem. Phys. 112, 8053 (2000).

[9] D. Blume and C. H. Greene, J. Chem. Phys. 113, 4242 (2000).

[10] J. C. Light and T. Carrington, in Advances in chemical physics, edited by I. Prigogine and S. A. Rice (Wiley, 2000), vol. 114, p. 263.

[11] X. G. Wang and T. Carrington, J. Phys. Chem. A 111, 10220 (2007).

[12] J. Sarka, C. Petty, and B. Poirier, J. Chem. Phys. 151, 174304 (2019).

[13] J. Avery, Hyperspherical Harmonics: Applications in Quantum Theory (Kluwer, 1989).

[14] Y. Suzuki, Phys. Rev. C 101, 014002 (2020).

[15] D. Wang and A. Kuppermann, J. Chem. Phys. 115, 9184 (2001).

[16] L. M. Delves, Nucl. Phys. 9, 391 (1958/59).

[17] L. M. Delves, Nucl. Phys. 20, 275 (1960).

[18] J. Dohet-Eraly and M. Viviani, Computer Physics Communications 253, 107183 (2020).

[19] M. Gattobigio, A. Kievsky, M. Viviani, and P. Barletta, Phys. Rev. A 79, 032513 (2009).

[20] A. Kuppermann, J. Phys. Chem. A 101, 6368 (1997).

[21] V. Aquilanti and S. Cavalli, J. Chem. Soc. Faraday Trans. 93(5), 801 (1997).

[22] W. Zickendraht, J. Math. Phys. 10, 30 (1969).

[23] W. Zickendraht, Annals of Physics 111, 162 (1978).

[24] R. G. Littlejohn, K. A. Mitchell, and V. Aquilanti, Phys. Chem. Chem. Phys. 1, 1259 (1999).

[25] V. Aquilanti, A. Beddoni, A. Lombardi, and R. G. Littlejohn, Int. J. Quant. Chem. 89, 277 
(2002).

[26] V. Aquilanti, A. Lombardi, and R. G. Littlejohn, Theor. Chem. Acc. 111, 400 (2004).

[27] B. Lepetit, D. Wang, and A. Kuppermann, J. Chem. Phys. 125, 133505 (2006).

[28] A. Kuppermann, J. Phys. Chem. 100, 2621 (1996).

[29] A. S. Davydov, Quantum Mechanics (Addison-Wesley, Reading, MA, 1965).

[30] M. Hamermesh, Group theory and its applications to physical problems (Pergamon Press, 1962).

[31] F. Karlický, B. Lepetit, R. Kalus, and F. X. Gadéa, J. Chem. Phys. 126, 174305 (2007).

[32] L. Velilla, B. Lepetit, A. Aguado, J. Beswick, and M. Paniagua, J. Chem. Phys. 129, 084307 (2008).

[33] F. Karlický, B. Lepetit, R. Kalus, I. Paidarová, and F. X. Gadéa, J. Chem. Phys. 128, 124303 (2008).

[34] F. Karlický, B. Lepetit, R. Kalus, and F. X. Gadéa, J. Chem. Phys. 134, 084305 (2011).

[35] O. Tolstikhin, S. Watanabe, and M. Matsuzawa, J. Phys. B : At. Mol. Opt. Phys. 29, L389 (1996).

[36] J. Light and T. Carrington, Adv. Chem. Phys. 114, 263 (2000).

[37] D. T. Colbert and W. H. Miller, J. Chem. Phys. 96, 1982 (1992).

[38] M. Tinkham, Group theory and quantum mechanics (McGraw-Hill, 1964).

[39] B. Yang, W. Chen, and B. Poirier, J. Chem. Phys. 135, 094306 (2011).

[40] J. Montgomery and B. Poirier, J. Chem. Phys. 119, 6609 (2003). 
TABLE I: $\mathrm{Ne}_{4}$ energy levels in unit of $\epsilon$ for the $\Gamma=A_{1}^{(3)}, \Pi=0$ and $\Gamma=A_{2}^{(3)}, \Pi=1$ calculations. The present results are compared with those of ref. [12] (Table VII). The IRREPs of $S_{4}$ to which each state belongs are also given. For each of the $T_{1}^{(4)}$ or $T_{2}^{(4)}$ states, 2 almost equal energies are given in ref. [12] (Table VII), only the lowest of both is given in the present Table. The present naming convention of the $T_{1}^{(4)}$ and $T_{2}^{(4)}$ IRREPs is different from the one used in ref. [12] (see section II D).

\begin{tabular}{|c|c|c|c|c|c|c|c|c|}
\hline & \multicolumn{4}{|c|}{$A_{1}^{(3)}, \Pi=0$} & \multicolumn{4}{|c|}{$A_{2}^{(3)}, \Pi=1$} \\
\hline & IRREP & $E$ (present) & $E_{\text {ref }}$ (ref. [12]) & $E-E_{r e f}$ & IRREP & $E$ (present) & $E_{\text {ref }}$ (ref. [12]) & $E-E_{r e f}$ \\
\hline 1 & $A_{1}^{(4)}$ & -3.454 & -3.4494 & -0.005 & $A_{2}^{(4)}$ & -3.454 & -3.4494 & -0.005 \\
\hline 2 & $T_{2}^{(4)}$ & -2.984 & -2.9844 & 0.000 & $T_{1}^{(4)}$ & -2.983 & -2.9830 & 0.000 \\
\hline 3 & $A_{1}^{(4)}$ & -2.961 & -2.9638 & 0.003 & $A_{2}^{(4)}$ & -2.957 & -2.9586 & 0.002 \\
\hline 4 & $T_{2}^{(4)}$ & -2.885 & -2.9073 & 0.022 & $T_{1}^{(4)}$ & -2.847 & -2.8591 & 0.012 \\
\hline 5 & $A_{1}^{(4)}$ & -2.867 & -2.8890 & 0.022 & $A_{2}^{(4)}$ & -2.808 & -2.8224 & 0.014 \\
\hline 6 & $T_{2}^{(4)}$ & -2.778 & -2.7913 & 0.013 & $T_{1}^{(4)}$ & -2.694 & -2.7093 & 0.015 \\
\hline 7 & $A_{1}^{(4)}$ & -2.733 & -2.7450 & 0.012 & $A_{2}^{(4)}$ & -2.677 & -2.6840 & 0.007 \\
\hline 8 & $A_{1}^{(4)}$ & -2.652 & -2.6573 & 0.005 & $A_{2}^{(4)}$ & -2.604 & -2.6158 & 0.012 \\
\hline 9 & $T_{2}^{(4)}$ & -2.605 & -2.6171 & 0.012 & $T_{1}^{(4)}$ & -2.539 & -2.5490 & 0.010 \\
\hline 10 & $A_{1}^{(4)}$ & -2.571 & -2.5836 & 0.012 & $A_{2}^{(4)}$ & -2.515 & -2.5249 & 0.010 \\
\hline 11 & $T_{2}^{(4)}$ & -2.529 & -2.5459 & 0.016 & $T_{1}^{(4)}$ & -2.494 & -2.5065 & 0.012 \\
\hline 12 & $A_{1}^{(4)}$ & -2.510 & -2.5243 & 0.014 & $T_{1}^{(4)}$ & -2.472 & -2.4862 & 0.014 \\
\hline 13 & $T_{2}^{(4)}$ & -2.494 & -2.5162 & 0.022 & $T_{1}^{(4)}$ & -2.431 & -2.4564 & 0.025 \\
\hline 14 & $T_{2}^{(4)}$ & -2.472 & -2.5090 & 0.037 & $A_{2}^{(4)}$ & -2.415 & -2.4291 & 0.014 \\
\hline 15 & $T_{2}^{(4)}$ & -2.458 & -2.4794 & 0.021 & $A_{2}^{(4)}$ & -2.404 & -2.4230 & 0.019 \\
\hline 16 & $A_{2}^{(4)}$ & -2.425 & -2.4473 & 0.022 & $T_{1}^{(4)}$ & -2.395 & -2.4221 & 0.027 \\
\hline
\end{tabular}


TABLE II: Row orthonormal hyperspherical coordinates $\left(\delta_{1}, \delta_{2}, \delta_{3}\right)$ for $R_{1}$ to $R_{6}$ rhombuses which are labelled $i-j k-l$, where $i, j, k, l$ are Ne atom numbers. Atoms $i$ and $l$ form the pair of opposite vertices connected by the longest diagonal, $j$ and $k$ by the shortest one. The angle $\alpha$ at vertices $i$ and $l$ is thus such that $\alpha \leq \pi / 2$. Calling $d$ the side of the rhombus, the hyperradius is given by $\rho=2^{\frac{5}{6}} d$ and the kinematic invariant angles by : $\theta_{R}=\alpha / 2$ and $\phi_{R}=0$ (coplanar configuration). In the Table $: \delta_{R}=\arcsin \left(\frac{1}{\sqrt{3}}\right) \approx 35^{\circ}$. The 4 configurations $i-j k-l, l-j k-i, i-k j-l$ and $l-k j-i$ correspond to the same set of coordinates $\left(\rho, \theta, \phi, \delta_{1}, \delta_{2}, \delta_{3}\right)$. Appendix A explains how these coordinates have been obtained.

\begin{tabular}{|c|c|c|}
\hline Rhombus \# & Configuration & $\delta_{1}, \delta_{2}, \delta_{3}$ \\
\hline $\mathrm{R}_{1}$ & $3-12-4$ & $\frac{\pi}{2}, \pi-\delta_{R}, \frac{\pi}{2}$ \\
$\mathrm{R}_{2}$ & $1-23-4$ & $\frac{\pi}{2}, \delta_{R}, \frac{5 \pi}{6}$ \\
$\mathrm{R}_{3}$ & $2-13-4$ & $\frac{\pi}{2}, \delta_{R}, \frac{\pi}{6}$ \\
$\mathrm{R}_{4}$ & $2-14-3$ & $\pi-\delta_{R}, \frac{\pi}{2}, \frac{\pi}{3}$ \\
$\mathrm{R}_{5}$ & $1-24-3$ & $\delta_{R}, \frac{\pi}{2}, \frac{2 \pi}{3}$ \\
$\mathrm{R}_{6}$ & $1-34-2$ & $\delta_{R}, \frac{\pi}{2}, 0$ \\
\hline
\end{tabular}


TABLE III: Row orthonormal hyperspherical coordinates $\left(\delta_{1}, \delta_{2}, \delta_{3}\right)$ for $T_{1}-T_{12}$ trigonal geometries. These configurations are labelled $i-j-k l$, where $i, j, k, l$ are Ne atom numbers. Atoms $j k l$ form an equilateral configuration and atom $i$ is bonded to the central atom $j$. Bond distances $j i$, $j k, j l$ and $k l$ are the same $(=d)$, however these trigonal configurations are not fully symmetric as $j i, j k$ and $j l, j i$ bond angles are $150^{\circ}$ whereas the $j k, j l$ bond angle is $60^{\circ}$. For all trigonal configurations of the Table, the hyperradius is given by $\rho=d \frac{(4+\sqrt{3})^{\frac{1}{2}}}{2^{\frac{1}{6}}}$ and the kinematic invariant angles $\theta$ and $\phi$ are given by : $\theta_{T}=\arcsin \left(\frac{1}{\sqrt{4+\sqrt{3}}}\right) \approx 25^{\circ}$ and $\phi_{T}=0$ (coplanar configuration). The values of the angles used in the Table are:

$\delta_{T}^{1}=\frac{\pi}{4}-\frac{\delta_{R}}{2}=\arcsin \left(\sqrt{\frac{1}{2}-\frac{1}{2 \sqrt{3}}}\right) \approx 27^{\circ}, \delta_{T}^{2}=3 \delta_{T}^{1}=\arccos \left(\sqrt{\frac{1}{2}-\frac{5}{6 \sqrt{3}}}\right) \approx 82^{\circ}$, $\delta_{T}^{3}=\arccos \left(-\sqrt{\frac{30}{37}+\frac{2 \sqrt{3}}{37}}\right) \approx 162^{\circ}, \delta_{T}^{4}=\arccos \left(\sqrt{\frac{1}{6}+\frac{1}{6 \sqrt{3}}}\right) \approx 59^{\circ}$, $\delta_{T}^{5}=\arctan \left(-\left(1+\frac{1}{\sqrt{3}}\right)\right) \approx 122^{\circ}$.

$\delta_{R}$ is defined in the caption of Table II. The 2 configurations $i-j-k l$ and $i-j-l k$ correspond to the same set of coordinates $\rho, \theta, \phi, \delta_{1}, \delta_{2}, \delta_{3}$. Appendix A explains how these coordinates have been obtained.

\begin{tabular}{|c|c|c|}
\hline Trigonal geom. \# & Configuration & $\delta_{1}, \delta_{2}, \delta_{3}$ \\
\hline $\mathrm{T}_{1}$ & $4-3-12$ & $\frac{\pi}{2}, \delta_{T}^{1}, \frac{\pi}{2}$ \\
$\mathrm{~T}_{2}$ & $4-2-13$ & $\frac{\pi}{2}, \pi-\delta_{T}^{1}, \frac{\pi}{6}$ \\
$\mathrm{~T}_{3}$ & $4-1-23$ & $\frac{\pi}{2}, \pi-\delta_{T}^{1}, \frac{5 \pi}{6}$ \\
$\mathrm{~T}_{4}$ & $3-4-12$ & $\frac{\pi}{2}, \delta_{T}^{2}, \frac{\pi}{2}$ \\
$\mathrm{~T}_{5}$ & $2-4-13$ & $\frac{\pi}{2}, \pi-\delta_{T}^{2}, \frac{\pi}{6}$ \\
$\mathrm{~T}_{6}$ & $1-4-23$ & $\frac{\pi}{2}, \pi-\delta_{T}^{2}, \frac{5 \pi}{6}$ \\
$\mathrm{~T}_{7}$ & $3-1-24$ & $\pi-\delta_{T}^{3}, \pi-\delta_{T}^{4}, \delta_{T}^{5}-\frac{\pi}{3}$ \\
$\mathrm{~T}_{8}$ & $2-1-34$ & $\pi-\delta_{T}^{3}, \delta_{T}^{4}, \pi-\delta_{T}^{5}$ \\
$\mathrm{~T}_{9}$ & $1-3-24$ & $\pi-\delta_{T}^{3}, \delta_{T}^{4}, \frac{5 \pi}{3}-\delta_{T}^{5}$ \\
$\mathrm{~T}_{10}$ & $3-2-14$ & $\delta_{T}^{3}, \pi-\delta_{T}^{4}, \frac{4 \pi}{3}-\delta_{T}^{5}$ \\
$\mathrm{~T}_{11}$ & $2-3-14$ & $\delta_{T}^{3}, \delta_{T}^{4}, \delta_{T}^{5}-\frac{2 \pi}{3}$ \\
$\mathrm{~T}_{12}$ & $1-2-34$ & $\delta_{T}^{3}, \delta_{T}^{4}, \delta_{T}^{5}$ \\
\hline
\end{tabular}



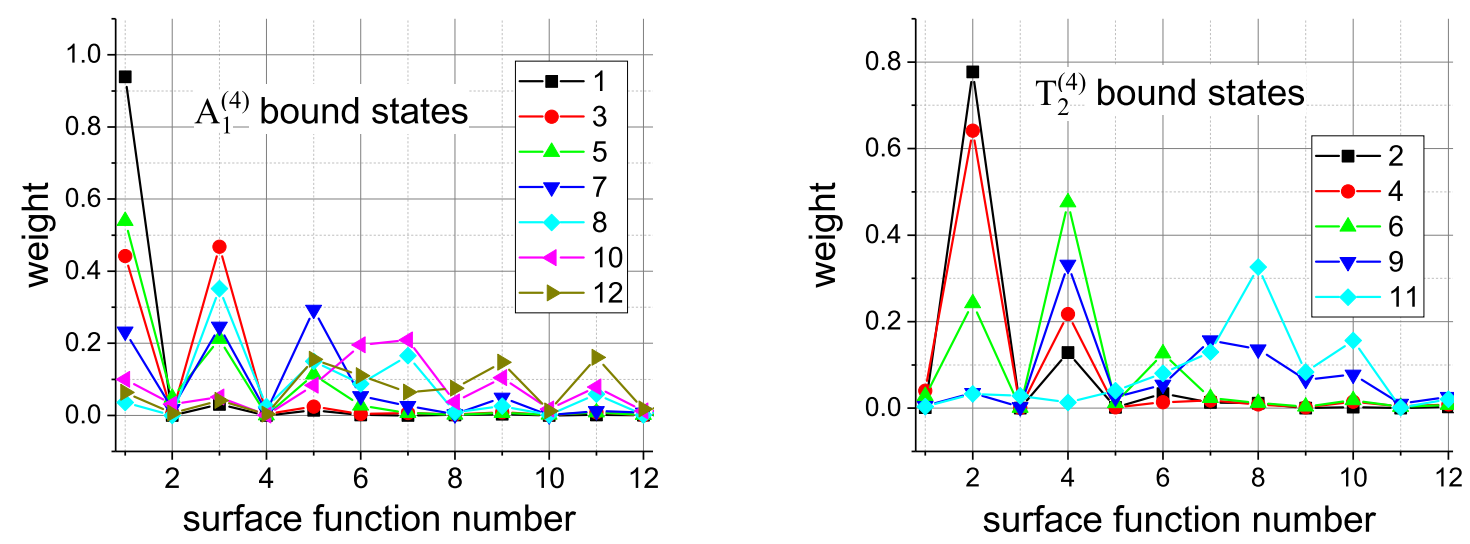

FIG. 1: Integrated weight of the surface function $\# \mathrm{i}\left(\Phi_{i}^{J M_{J} \Pi \Gamma}\right)$ in the vibrational state \# $\mathrm{k}$

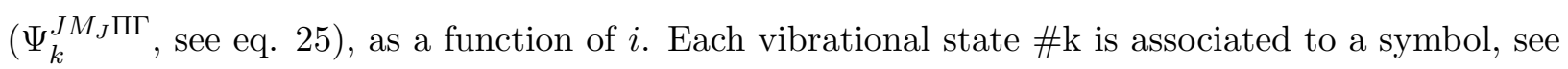
the insets of the figures. Left : $\Pi=0$ parity, $\mathrm{A}_{1}^{(4)}$ permutation symmetry. Right : $\Pi=0, \mathrm{~T}_{2}^{(4)}$. 


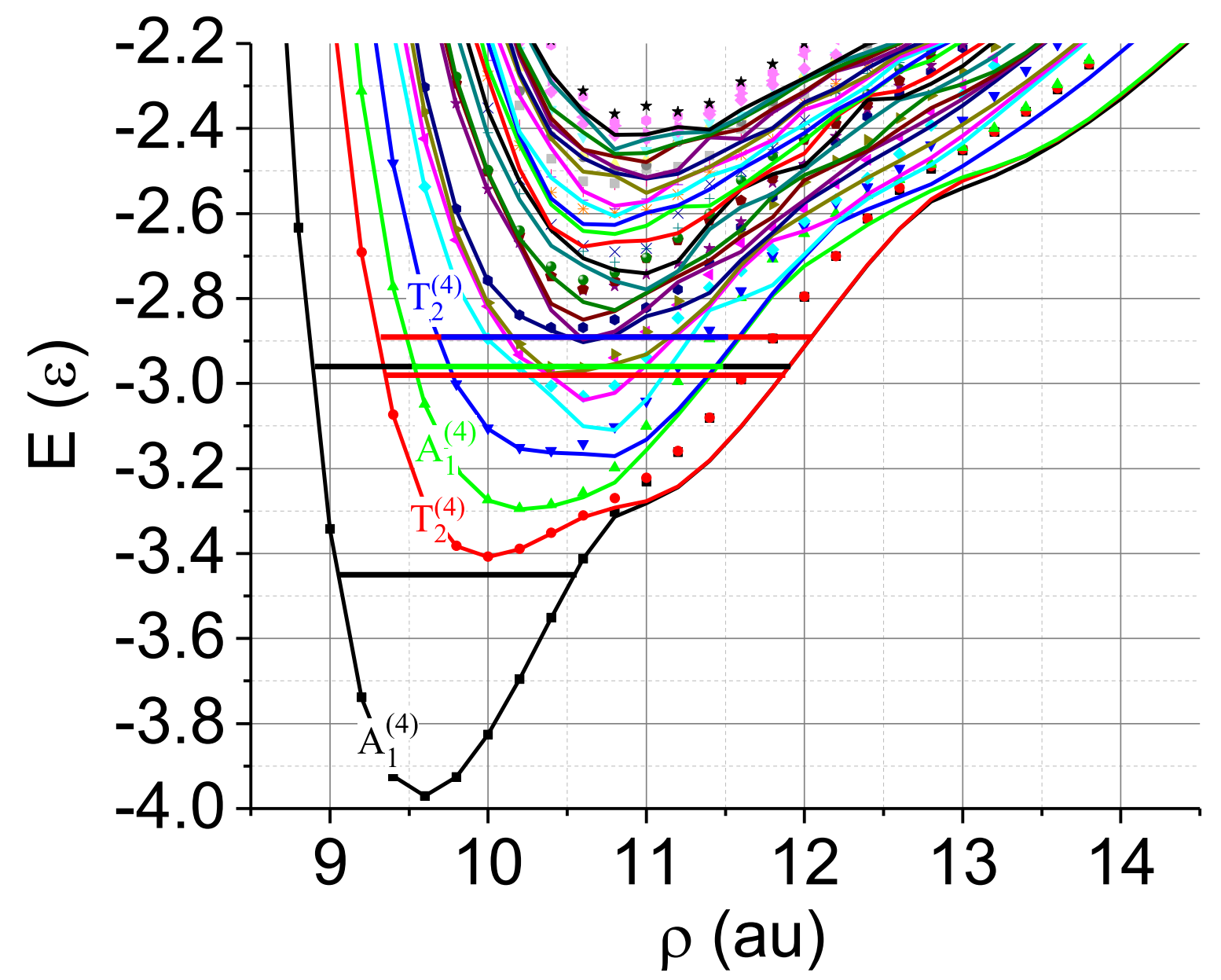

FIG. 2: Surface function energies (unit : $\epsilon$, the $\mathrm{Ne}_{2}$ pair-potential well depth) as a function of the hyperradius (atomic unit). The 0-energy corresponds to 4-body dissociation. They result from 2 calculations, $\Gamma=A_{1}^{(3)}, \Pi=0$ (full lines) and $\Gamma=A_{2}^{(3)}, \Pi=1$ (symbols). $\mathrm{S}_{4}$ symmetry labels are shown for the four lowest potentials. The 4 lowest bound state energies $(\Pi=0)$ are shown as horizontal segments. The lowest energy bound state (for each $A_{1}^{(4)}$ and $T_{2}^{(4)}$ symmetry) is well represented by the adiabatic approximation and is supported almost entirely by a single surface function potential, the color of segment is the one of the corresponding potential. As at least 2 surface functions are necessary in the expansion of the first excited states (in each symmetry), their energies are represented by segments with 2 colors identical to those of the corresponding potentials. 


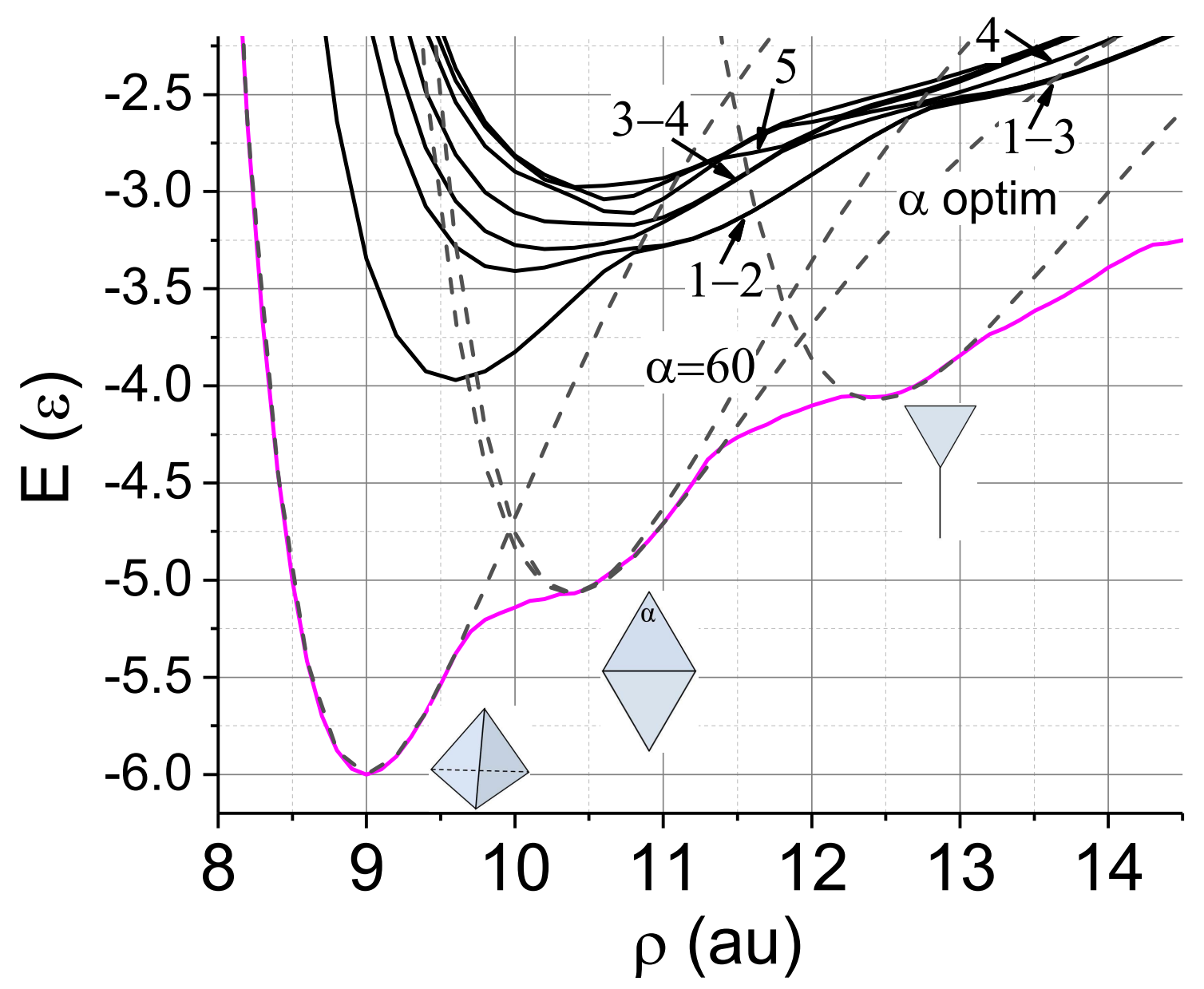

FIG. 3: Potential energy minimum (blue continuous line) as a function of $\rho$. The 0-energy corresponds to 4-body dissociation. The dashed lines correspond to the potential energies for selected geometries of the system : tetrahedron, rhombus (vertex angle fixed : $\alpha=\frac{\pi}{3}$ or optimized to minimize potential), trigonal (bond angles with respect to the central atom : $\frac{5 \pi}{6}, \frac{5 \pi}{6}, \frac{\pi}{3}$ ). These geometries correspond to the potential minimum in the vicinity of $\rho=8.998 \mathrm{au}, \rho=10.390 \mathrm{au}$ and $\rho=12.438 \mathrm{au}$, respectively. The lowest $\Pi=0 A_{1}^{(4)}$ and $T_{2}^{(4)}$ surface function energies are also shown as black continuous lines. The excitation numbers of the surface functions plotted on Fig. 4-7 are also indicated. 

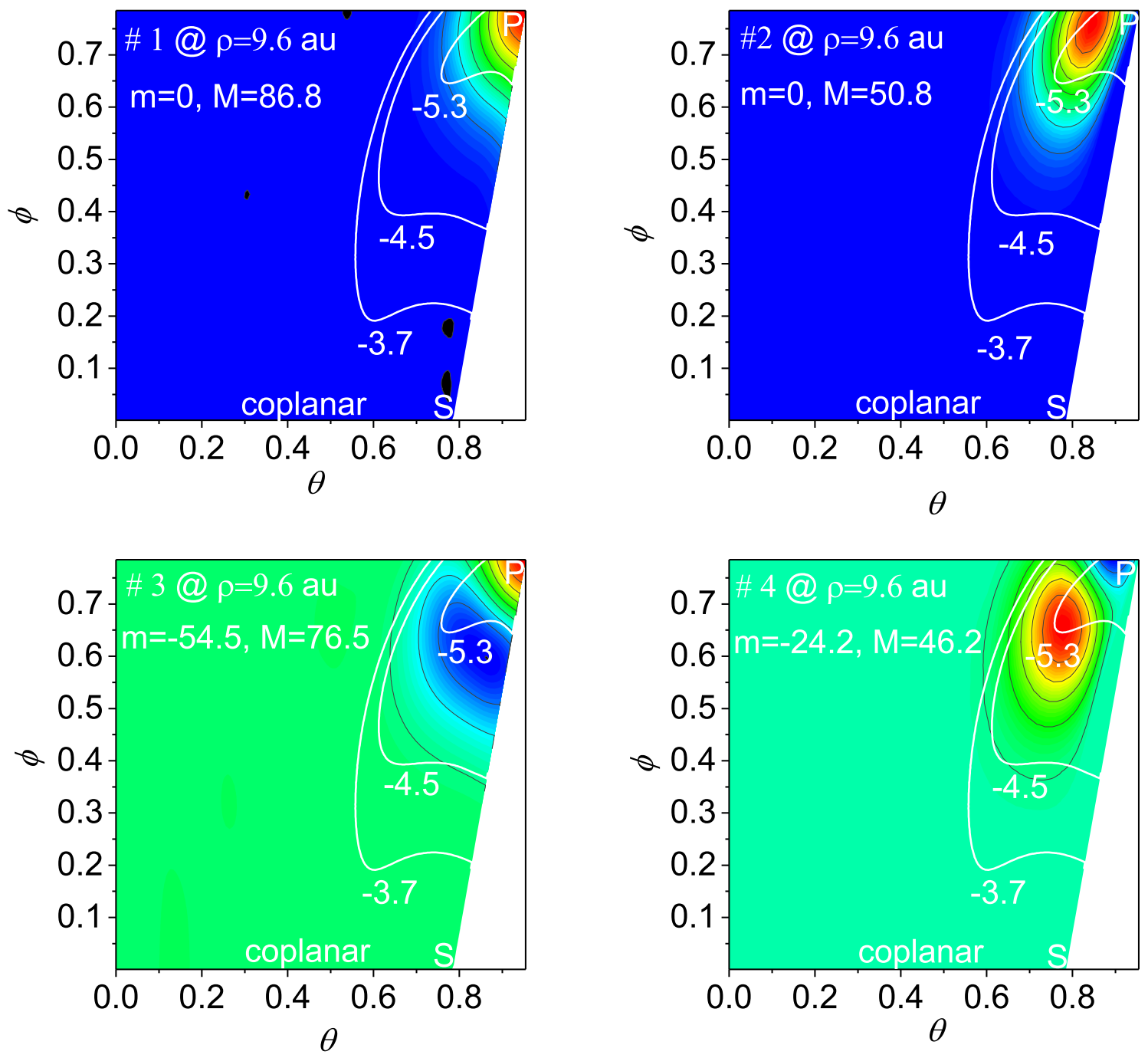

FIG. 4: Surface function amplitude color plots for the ground and excited surface functions, $\Pi=0$. The point labelled $\mathrm{P}$ corresponds to the tetraedral (Pyramid) configuration, the point $\mathrm{S}$ to coplanar symmetric top configurations and the line $\phi=0$ to all coplanar configurations. The color scale gives the amplitude from blue (lowest amplitude, given by the value of $\mathrm{m}$ on the graph) to red (max amplitude, given by the value of $\mathrm{M}$ on the graph). Results are shown in the $(\theta, \phi)$ plane for $\rho=9.6 \mathrm{au}, \delta_{1}=\delta_{2}=\delta_{3}=\frac{\pi}{2}$. The number shown in the upper left corner is the excitation number, starting with \# 1 for the ground state. States \#1 an \# 3 belong to the $A_{1}^{(4)}$ IRREP, states \# 2 and \# 4 to $T_{2}^{(4)}$. The white lines are the contour plots of the potential $-5.3,-4.5,-3.7 \epsilon$. 

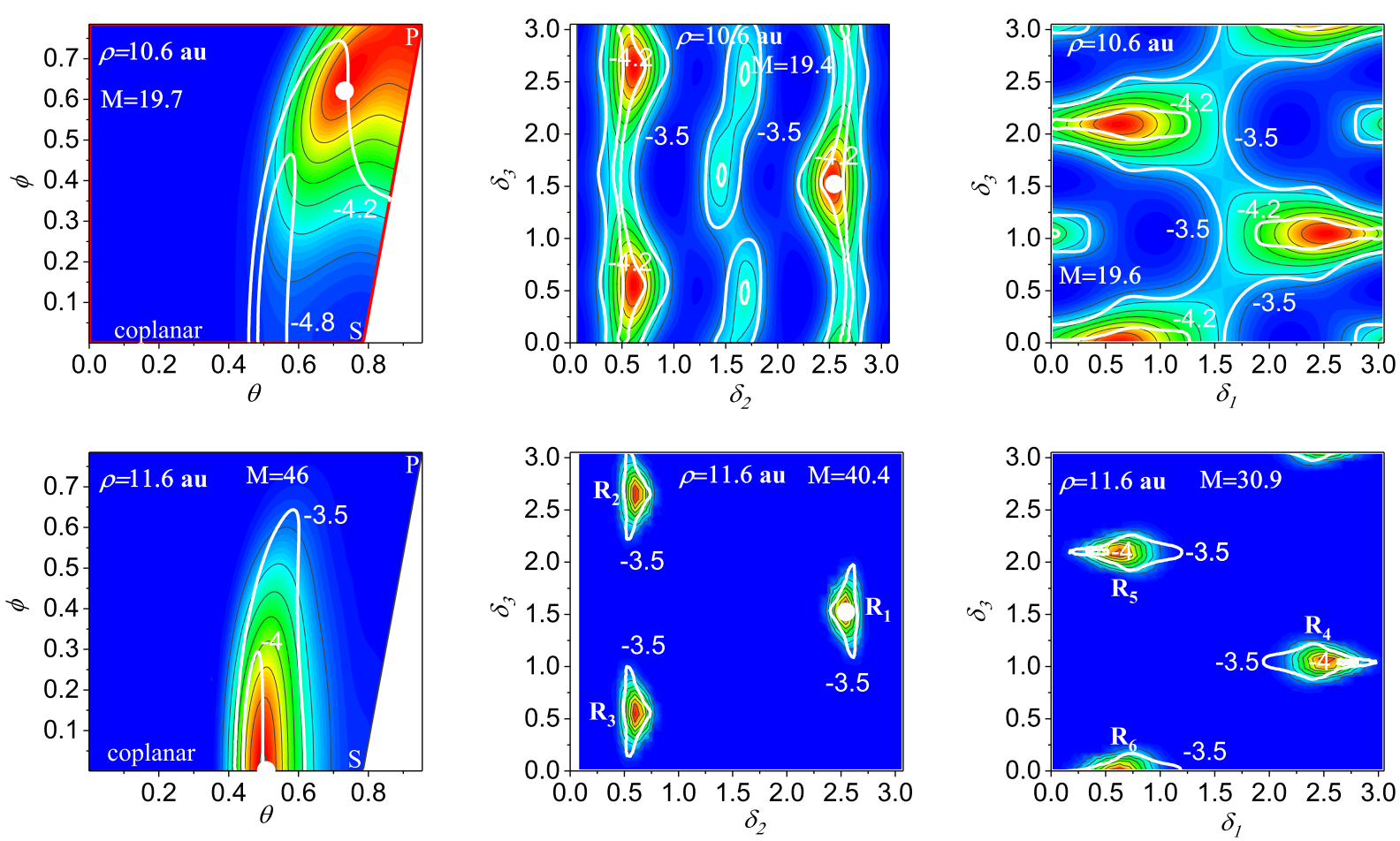

FIG. 5: Surface function amplitude color plots for the ground state surface function, $\Pi=0\left(A_{1}^{(4)}\right.$ permutation symmetry). The color scale gives the amplitude from blue (negligible amplitude) to red (max amplitude, given by the value of $\mathrm{M}$ on the graph). The white lines are contour plots of the potential with labels in units of $\epsilon$. From top to bottom : $\rho=10.6,11.6$ au.

Left : plots in the $(\theta, \phi)$ plane for fixed values of the other coordinates : $\delta_{1}=\frac{\pi}{2}, \delta_{2}=\pi-\delta_{R}$, $\delta_{3}=\frac{\pi}{2}$. See the caption of Table II for the definition of $\delta_{R}$. The point labelled $\mathrm{P}$ corresponds to the tetraedral (Pyramid) configuration, the point $\mathrm{S}$ to coplanar symmetric top configurations and the line $\phi=0$ to all coplanar configurations.

Center : plots in the $\left(\delta_{2}, \delta_{3}\right)$ plane for $\delta_{1}=\frac{\pi}{2}$. For $\rho=10.6 \mathrm{au}, \theta=0.73, \phi=0.62$. For $\rho=11.6$ $\mathrm{au}, \theta=\frac{\pi}{6}$ and $\phi=0$.

Right : plots in the $\left(\delta_{1}, \delta_{3}\right)$ plane. For $\rho=10.6 \mathrm{au}, \delta_{2}=\frac{\pi}{2}, \theta=0.73, \phi=0.62$. For $\rho=11.6 \mathrm{au}$, $\delta_{2}=\frac{\pi}{2}, \theta=\frac{\pi}{6}$ and $\phi=0$.

A thick white bullet in a $(\theta, \phi)$ plot (left frame) indicates the values of $\theta$ and $\phi$ chosen for the plots in the $\left(\delta_{2}, \delta_{3}\right)$ planes at the same $\rho$. Similarly, a thick white bullet in a $\left(\delta_{2}, \delta_{3}\right)$ plot (middle frame) indicates the values of $\delta_{2}$ and $\delta_{3}$ used in the corresponding $(\theta, \phi)$ plot. The labels $\mathrm{R}_{1}-\mathrm{R}_{6}$ correspond to the coordinates listed in Table II. 

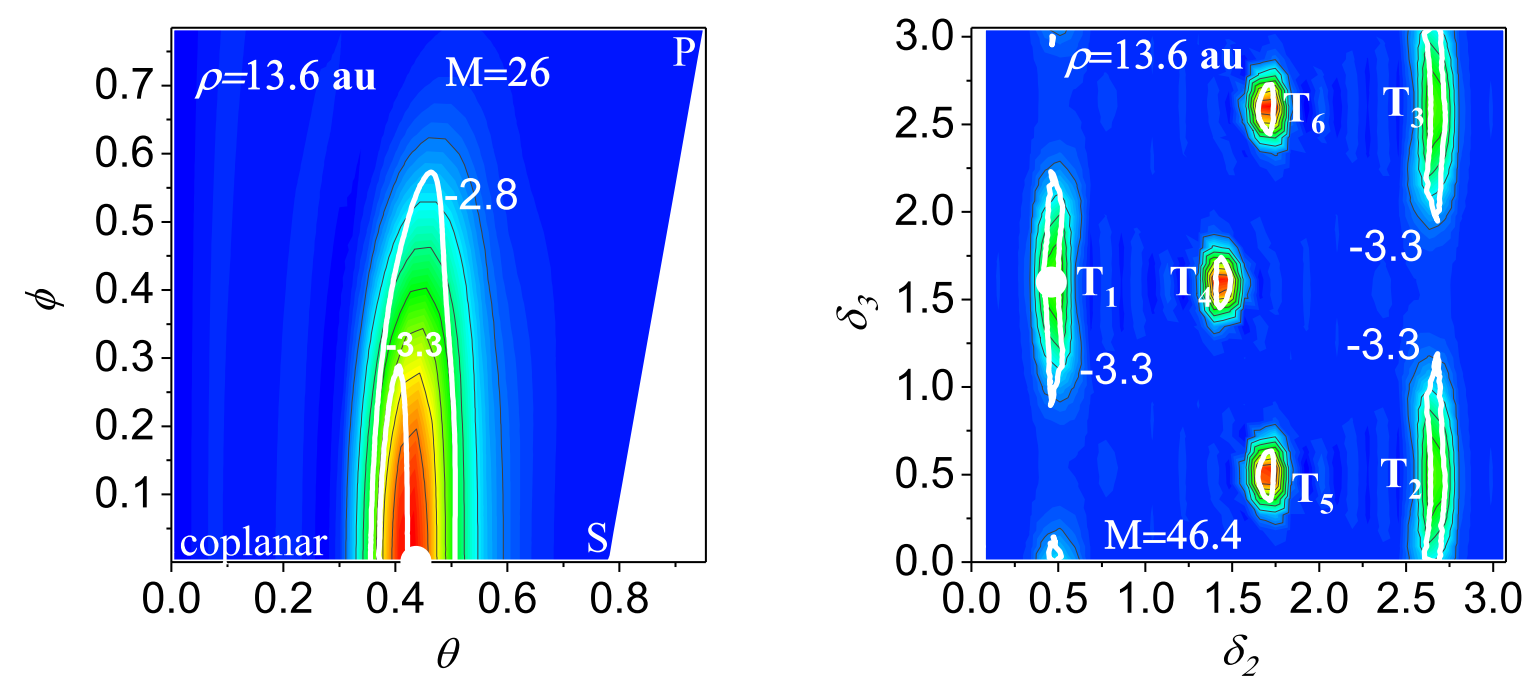

FIG. 6: Surface function amplitude color plots for the ground state surface function for $\Pi=0\left(A_{1}^{(4)}\right.$ permutation symmetry) at $\rho=13.6 \mathrm{au}$. The color scale gives the amplitude from blue (negligible amplitude) to red (max amplitude, given by the value of $M$ on the graph). The white lines are contour plots of the potential with labels in units of $\epsilon$.

Left : plot in the $(\theta, \phi)$ plane for fixed values of the other coordinates : $\delta_{1}=\frac{\pi}{2}, \delta_{2}=\delta_{T}^{1}, \delta_{3}=\frac{\pi}{2}$. The point labelled $\mathrm{P}$ corresponds to the tetraedral (Pyramid) configuration, the point $\mathrm{S}$ to coplanar symmetric top configurations and the line $\phi=0$ to all coplanar configurations.

Right : plot in the $\left(\delta_{2}, \delta_{3}\right)$ plane for $\delta_{1}=\frac{\pi}{2}, \theta=\theta_{T}, \phi=0$.

See the caption of Table III for the definitions of $\delta_{T}^{1}$ and $\theta_{T}$. A thick white bullet in the $(\theta, \phi)$ plot (left frame) indicates the values of $\theta$ and $\phi$ chosen for the plots in the $\left(\delta_{2}, \delta_{3}\right)$ plane. Similarly, a thick white bullet in the $\left(\delta_{2}, \delta_{3}\right)$ plot (right frame) indicates the values of $\delta_{2}$ and $\delta_{3}$ used in the corresponding $(\theta, \phi)$ plot. The labels $\mathrm{T}_{1}-\mathrm{T}_{6}$ correspond to the coordinates listed in Table III. 

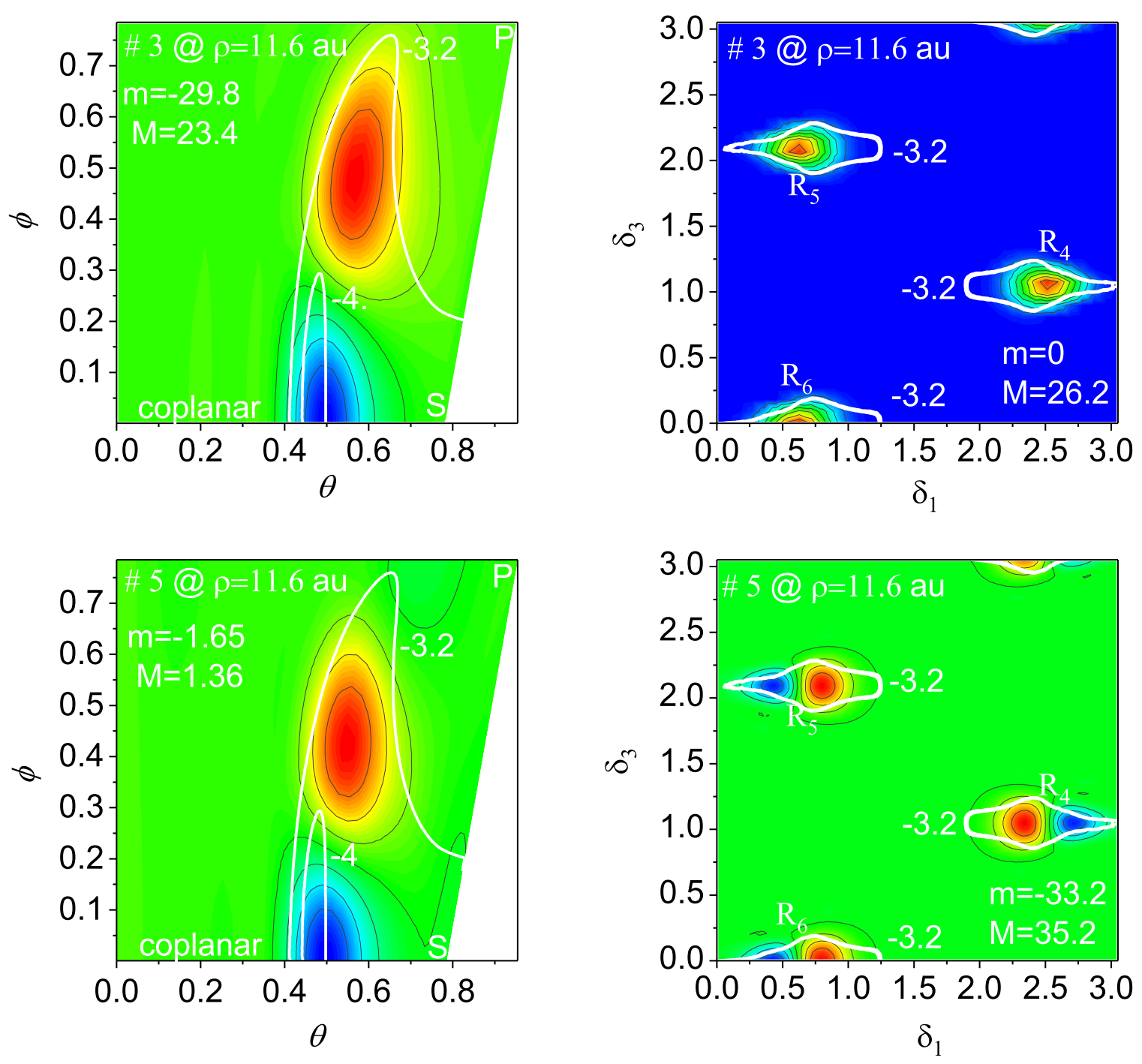

FIG. 7: Surface function amplitude color plots for excited states surface function for $\rho=11.6 \mathrm{au}$ $\Pi=0$. The color scale gives the amplitude from blue (lowest amplitude, given by the value of $\mathrm{m}$ on the graph) to red (max amplitude, given by the value of $\mathrm{M}$ on the graph). The number shown in the upper left corner is the excitation number (see Fig. 3), starting with \# 1 for the ground state. The white lines are contour plots of the potential with labels in units of $\epsilon$. Left : results in the $(\theta, \phi)$ plane, $\delta_{1}=\frac{\pi}{2}, \delta_{2}=\pi-\delta_{R}, \delta_{3}=\frac{\pi}{2}$. See the caption of Table II for the definition of $\delta_{R}$. The point labelled $\mathrm{P}$ corresponds to the tetraedral (Pyramid) configuration, the point $\mathrm{S}$ to coplanar symmetric top configurations and the line $\phi=0$ to all coplanar configurations. Right : results in the $\left(\delta_{1}, \delta_{3}\right)$ plane, $\theta=\frac{\pi}{6}, \phi=0, \delta_{2}=\frac{\pi}{2}$. 